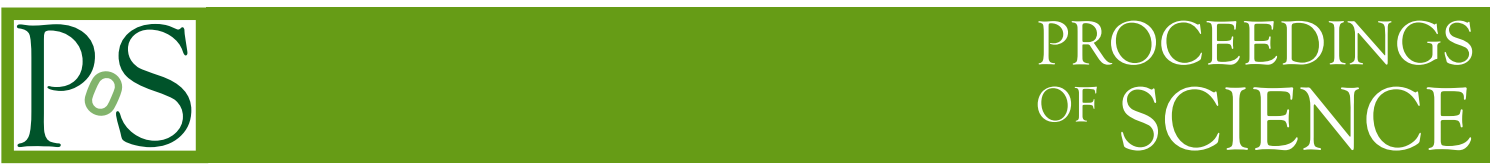

\title{
Intermediate Mass Black Holes: A brief review
}

\author{
Filippos Koliopanos* \\ CNRS, IRAP, 9 Av. colonel Roche, BP 44346, F-31028 Toulouse cedex 4, France \\ Université de Toulouse; UPS-OMP; IRAP, Toulouse, France \\ E-mail: tEkoliopanos@irap.omp.eu
}

Intermediate mass black holes (IMBHs) are an (as yet) elusive class of black holes that are expected to lie in the $10^{2}-10^{5} M_{\odot}$ range, between the firmly established stellar-mass black holes and $\gtrsim 10^{6} M_{\odot}$ supermassive black holes. Predicted by a variety of theoretical models, IMBHs are the potential seeds of supermassive black holes and are expected to power some of the brightest extra-nuclear X-ray sources. This brief review is the result of a presentation and subsequent discussion of IMBHs that took place during the 12th International Frascati workshop on "Multifrequency Behaviour of High Energy Cosmic Sources". The manuscript aims to provide a concise and up-to-date review of the different evolutionary scenarios for the creation of IMBHs. Throughout the text I emphasize the importance of the identification and classification of IMBHSs in our effort to understand the formation of supermassive black holes and their co-evolution with their host galaxies.

XII Multifrequency Behaviour of High Energy Cosmic Sources Workshop 12-17 June, 2017

Palermo, Italy

\footnotetext{
*Speaker.
} 


\section{Introduction}

First theorized by Schwarzschild (1916) and reformulated by Droste (1917) [U], Hilbert (1917) [] and Weyl (1917) [[] as peculiar repercussions of Einstein's theory of gravitation, black holes

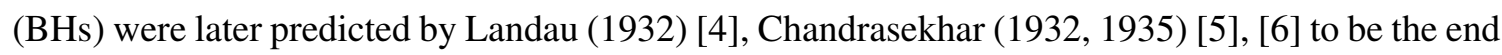
point of massive stars that collapse into singularities. However, it wasn't until 1972 and the dynamical estimation of mass of several $M_{\odot}$ for the "dark" companion of the Cyg X-1 binary star system, that an astrophysical source was firmly recognized as black hole (BH) [प]. Numerous subsequent observations of BH X-ray binaries (BH-XRBS: e.g. [8, Q Q] ) have established the abundance of stellar mass BHs in the Universe, leading to the confirmation of BH mergers, in the recent discovery of gravitational waves [ए人, ए], ए2].

In the 1970s Sanders and Lowinger (1972) [[3] used near infrared observations of the galactic center to infer the presence of a massive central object exceeding $10^{5} M_{\odot}$, while dynamical estimations suggested the presence of a supermassive black hole (SMBH) in the center of M87. Indeed Rees in 1984 [[4] promoted the presence of SMBHs in the centers of massive galaxies. Approximately a decade later an abundant accumulation of solid observational evidence for the presence of SMBHs in active galactic nuclei (beginning with the remarkable detection of a relativistically broadened $\mathrm{Fe} \mathrm{K} \alpha$ line in MCG-6-30-15 [ए5]], $\mathrm{H}_{2} \mathrm{O}$ maser emission from a $4 \times 10^{7} M_{\odot} \mathrm{SMBH}$ in NGC 4258 [ए]]), dynamical estimations of the SMBH in the center of the Milky Way (e.g. [ए]]), along with strong indications for weakly accreting SMBHs in most nearby galaxies, has convinced the vast majority of the astrophysics community that SMBHs lie in the centers of most - if not all - galaxies.

The wealth of observational confirmations for the existence of smaller mass BHs ( $\left.\lesssim 200 M_{\odot}\right)$ and SMBHs $\left(\gtrsim 10^{6} M_{\odot}\right)$ stands in stark contrast to the scarcity of observational evidence in favor of the existence of BHs between these two mass regimes. With the exception of very few strong candidates (see Section 4) and a modest sample of potential candidates, we have yet to confirm the existence intermediate mass black holes (IMBHs) - which are defined as BHs with $100 M_{\odot} \lesssim M_{\mathrm{BH}} \lesssim 10^{6} M_{\odot}-$ and are even further from establishing any characteristics of their population (i.e. the existence of two sub-populations of 100-1000 $M_{\odot}$ and $10^{4}-10^{5} M_{\odot}$ ). Establishing these facts is a crucial and necessary step in understanding stellar and galactic evolution as well as probing $\mathrm{BH}$ feedback in different mass regimes.

In terms of stellar evolution, IMBHs are the expected relics of evolved Population III (Pop III) stars. Produced inside $10^{6} M_{\odot}$ dark matter mini-haloes at redshift $\mathrm{z}>15$ (e.g. [108, ㅁ]), Pop III stars can exceed $200 M_{\odot}$. Their high mass is the result of the lack of heavier elements in their progenitor cloud which maintains low fragmentation, as the gas cools inefficiently and the Jeans mass is higher. The resulting massive Pop III stars retain their mass as they exhibit diminished stellar winds than what is expected in massive metal rich stars (e.g. [20, 221, 22, 221, 24, 25, 20]). After their short lifetime ( $\lesssim 3 \mathrm{Myr}$ ) Pop III stars may collapse into BHs that can exceed $200 M_{\odot}$ (e.g. [L7, [28, [29]). Discovery of IMBHs, in the $60-300 M_{\odot}$ range, will provide tremendous insight into the Pop III stars that as of yet cannot be observed directly ${ }^{1}$. Furthermore, IMBHs are also predicted to exist in accretion disks of active galactic nuclei (AGN, e.g. [3], B2], 33]) and produced

\footnotetext{
${ }^{1}$ Due to their large distance and low surface number densities Pop III stars will most likely be outside the reach of the upcoming James Webb Telescope [B]]
} 
by repeated mergers of compact remnants of massive star in circumnuclear giant $\mathrm{H}$ II regions with a dense stellar population (e.g. [34] or onto other massive stars in young massive clusters (e.g. [357, 136, 37, 38, 339]).

In terms of galaxy evolution, the detection - and more importantly - the determination of the spatial and mass distribution and the size of an IMBH population is a pivotal step in our understanding of formation of SMBHs and consequently galaxy formation itself. The discovery of luminous $\left(\mathrm{L} \gtrsim 10^{47} \mathrm{erg} / \mathrm{sec}\right.$ ) quasars at $\mathrm{z} \sim 6$ reveals that SMBHs with $M_{\mathrm{BH}} \gtrsim 10^{9} M_{\odot}$ must have formed within $\lesssim 1$ Gyr. This realization raises crucial questions with regard to the initial seeds of SMBHs and the process by which they are formed. The different formation paths will have a decisive impact on the star formation of the host galaxies. They can be divided into two main scenarios (that involve the presence of IMBHs) involving "light" or massive seeds. For a detailed review of the different formation paths of SMBHs see Volonteri et al. 2010 [40].

In the light seed scenario, the SMBH forms either via (super-Eddington) accretion onto $\sim 100 M_{\odot}$ IMBHs (e.g. [4], 42], 43], 44]]), or onto $\sim 10^{3} M_{\odot}$ IMBHs seeds which in turn are the result of multiple mergers of Pop III IMBH primordial X-ray binaries (e.g. [27, 45], 46]). In the massive seed or direct collapse - scenario the SMBH seed is the result of direct collapse of primordial pristine hydrogen gas in Ly $\alpha$ cooling haloes with virial temperatures exceeding $10^{4} \mathrm{~K}$. In addition to the lack of metal line cooling, the halo requires the presence of strong Lyman-Werner UV radiation to destroy molecular hydrogen and prevent early collapse and fragmentation, until the structure collapses into an IMBH of $\sim 10^{4} M_{\odot}$ (e.g. [47, 48, 49, 50] ). Depending on the mass of the seed different evolutionary paths lead to the creation of the SMBH, which may involve sustained Eddington limited accretion or episodic super- Eddington accretion (e.g. Pacucci et al. 2015; Inayoshi et al. 2016). More importantly, the energy range of the resulting X-ray emission differs considerably, which in turn may result in positive or negative feedback on the formation of early galaxies. Hard X-rays (energies $\gtrsim 1 \mathrm{keV}$ ) are more penetrative and may heat the intergalactic medium, suppressing star formation, while soft X-rays $(0.1-1 \mathrm{keV}$ : emitted from more massive accreting IMBHs accreting at lower rates) can promote the formation of $\mathrm{H}_{2}$ by increasing the fraction of free electrons via photoionization, which in turn facilitates the cooling and collapse of gas, therefore promoting star-formation (e.g. [15], 52, [2], [44, [5], 56, 52, 58] ). Therefore, discovering and measuring the mass of a potential IMBHs population is a major step in understanding stellar evolution and galaxy formation at different mass regimes. The "relics" of the different formation paths are presented in an illustrative plot by Mezcua (2017)([5]], Figure 1 in this article. See also Fig.1 in Greene 2012) in her recent review of IMBHs. In addition to the different "sizes" of IMBHs predicted by the two scenarios, the direct collapse scenario predicts a much lower occupation fraction in low-mass galaxies than the Pop III remnant scenario [6]] (but also see Shirakata et al. 2016 implications [6]).

In addition to central SMBHs, primordial $\mathrm{BH}$ formation scenarios based on phase transitions in the early Universe (e.g. [62, 63, 64]) also predict the existence of IMBHs in galactic halos at a substantial distance from the galactic center [65], 66, 67]. While a considerable fraction of primordial IMBHs may merge to produce central SMBHs, it is likely that a considerable fraction of them, will remain in the interemdiate mass regime forming a population of IMBHs in the galactic halos. Members of such a population may manifest themselves as hyper-luminous X-ray sources, if they accrete matter from a companion (e.g. ESO 243-49 HLX-1: [68], see relevant section on 
ultraluminous X-ray sources below).

Below, I will present a concise review of the different observational methods employed in the search for IMBHs and their current observational constraints. For an extensive, recent review on IMBHs, see Mezcua 2017 [5]].

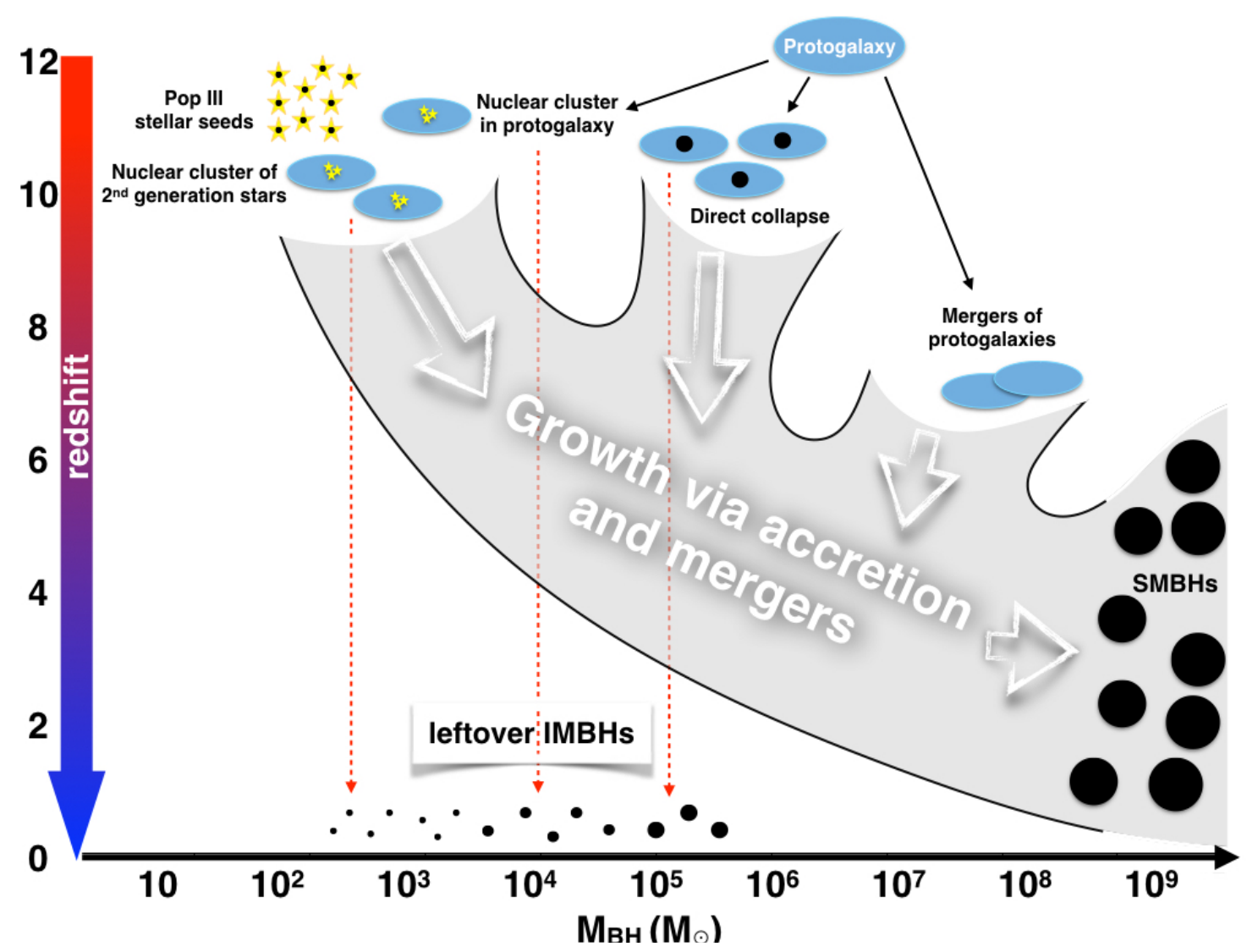

Figure 1: From Mezcua (2017), with permission from the author and IJMPD: Formation scenarios for IMBHs. Seed BHs in the early Universe could form from Population III stars, from mergers in dense stellar clusters formed out either from the second generation of stars or from inflows in protogalaxies, or from direct collapse of dense gas in protogalaxies, and grow via accretion and merging to $10^{9} M_{\odot}$ by z $\sim 7$. SMBHs could also directly form by mergers of protogalaxies at $\mathrm{z} \sim 6$. Those seed BHs that did not grow into SMBHs can be found in the local Universe as leftover IMBHs.

\section{Direct observational methods}

The most reliable method of detecting massive BHs (henceforth the term MBH will be used to refer to massive central BHs that may be either in the SMBH or the IMBH range) in the center of galaxies involves direct kinematic measurements. Such methods involve the dynamical measurements of individual sources, as is the case for Sgr A* ([ए], [9, 四, 四]), direct monitoring of gas motion orbiting the central $\mathrm{MBH}$ (e.g. by measuring the offset of $\mathrm{H}_{2} \mathrm{O}$ megamaser emission lines [ए]] or by 2D modeling of the nuclear gas kinematics using emission line imagery from high

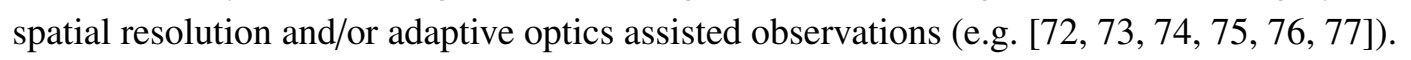


The radius of influence of the $\mathrm{BH}$ can be expressed in terms of the velocity dispersion of the

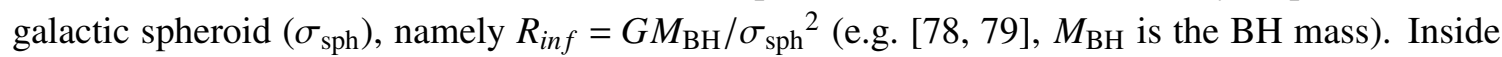
the sphere of influence, objects are expected to follow Keplerian orbits under the gravitational force of the BH. For a BH mass of $\sim 10^{5} M_{\odot}$ and $\sigma_{\text {sph }}=30 \mathrm{~km} / \mathrm{s}$ the radius of influence would be $0.5 \mathrm{pc}$, making it impossible to resolve - even for the nearest galaxies - using current telescopes. As such, so far there are no definitive direct estimations of IMBHs, even for the nearest candidates. The nearby dwarf galaxy NGC 4395 (see Section 4) hosts one of the lowest directly measured BH masses, with a central MBH of $\log M_{\mathrm{BH}}=5-6 M_{\odot}$, based on two-dimensional gas kinematic data [ख0]. This places the MBH in NGC 4395 at the lower end of SMBHs and tentatively in the IMBH range. Dynamical gas modeling in NGC 404 yielded an estimation of $\sim 10^{5} M_{\odot}[\mathrm{[}]$ ], however this estimation is dependent on underlying model assumptions and is strained by uncertainties in the central stellar mass profile. Only upper limits are provided for a handful more candidates (e.g. [82], [83, [84])

Another direct approach involves the reverberation mapping technique. Reverberation mapping involves accreting BHs and relies on the fact that the continuum emission of AGN is reprocessed in the high velocity photo-ionized gas that envelopes the central source - known as the broad line region (BLR). Since the continuum emission is variable, time delays between fluctuations observed in the continuum and fluctuations of the velocity broadened emission lines of the BLR provides an estimate of the distance between the BH and the BLR, assuming light travel time. The Doppler broadening of the lines can be used to infer the velocity of the orbiting material in the BLR, yielding an estimation for the mass of the central BH assuming Keplerian rotation (for a detailed review of the method see e.g. Peterson 2014 [85]]). More precisely the value that is measured has dimensions of mass and is known as the virial product $M_{v i r}=c \tau v^{2} / G$, where $\tau$ is the time lag and $v$ is the velocity of the material in the BLR. The actual mass of the BH is related to $M_{v i r}$ by a factor of order unity, known as the virial factor (f), which is introduced by the kinematics and geometry of the BLR.

In order to estimate the value of the virial factor, it is assumed that the $M_{\mathrm{BH}}$ values estimated by the reverberation mapping technique also follow the $M_{\mathrm{BH}}-\sigma_{\mathrm{sph}}$ distribution ( [86, 87], see Section 3). The assumption is that for a given value of the $\sigma_{\mathrm{sph}}$ the $M_{\mathrm{BH}}$ estimated with the reverberation technique must be equal (within the relation's inherent scatter) with the value predicted using the dynamically estimated $M_{\mathrm{BH}^{-}} \sigma_{\text {sph }}$ relation and thus the virial factor is estimated for each source (e.g. [ [88, 89, Q0], Q], Q2]]). Therefore any potential issues with the validity of the $M_{\mathrm{BH}}{ }^{-} \sigma_{\text {sph }}$ results (e.g. in the low-mass regime, see Section 3) will carry over to the virial mass estimations. Furthermore, recent analysis reveals that $M_{\mathrm{BH}}$ values derived from the accretion disk properties versus the virial mass estimates differ by a factor that is inversely proportional to the width of the broad emission lines, leading to significant viral mass mis-estimations [03]. An example of the consequences of different modeling assumptions are evident in the mass estimation of the IMBH candidate in NGC 4395 using reverberation mapping. Edri et al. (2012) measure a value of $M_{\mathrm{BH}}=(4.9 \pm 2.6) \times 10^{4} M_{\odot}$ [Q4]], placing it decisively in the IMBH range, but Peterson et al. (2005) estimate the mass to be much higher, at $\sim 3.6 \times 10^{5} M_{\odot}$ [Q25]. 


\section{Indirect observational methods}

Direct observing methods are limited to only the most nearby galaxies and are vulnerable due to modeling assumptions. Furthermore, the reverberation mapping method requires multi epoch observations in order to measure the spectral time lag. In order to probe further and - most importantly - less luminous and less massive candidates, a variety of indirect methods can be employed.

\subsection{IMBHs in AGN: Empirical relations between emission lines and/or optical continuum}

Virial mass estimates of central MBHs can be inferred using single epoch observations by making use of the empirical relation between optical AGN luminosity at $5100 \AA$ and the size of the BLR, namely $R_{B L R} \sim L_{5100} 0.7$ [Q6]]. The virial mass can then be estimated by inferring the velocity of the BLR using the broadening of the $\mathrm{H} \beta$ emission line (e.g., [07, 08, 09, ㅁ00, ए0]]). Furthermore, by demonstrating a clear linear relation between the $\mathrm{H} \alpha$ luminosity and the optical continuum luminosity and a correlation between the widths of the $\mathrm{H} \alpha$ and $\mathrm{H} \beta$ lines, Greene \& Ho (2005) were able to infer the virial mass system based solely on observations of the broadened $\mathrm{H} \alpha$ emission line. By way of optical spectroscopy and the use of classification techniques such as the "Baldwin, Phillips \& Terlevich" (BPT) diagrams [102] (which distinguish between LINERs, H II regions and AGN by measuring $[\mathrm{O} \mathrm{III}] / \mathrm{H} \beta,[\mathrm{N} \mathrm{II}] / \mathrm{H} \alpha$, and $[\mathrm{S} \mathrm{II}] / \mathrm{H} \alpha$ flux ratios), AGN are recognized among galactic surveys and their virial mass can be estimated by the above methods. This methodology has revealed more than 150 candidates with a central $\mathrm{MBH}$ in the $10^{5} M_{\odot} \lesssim M_{\mathrm{BH}} \lesssim 10^{6} M_{\odot}$ range (e.g. [103], [04]).

\subsection{Central IMBHs: Black hole mass - host bulge scaling relations}

Discovered simultaneously by two groups, Ferrarese \& Merritt 2000 and Gebhardt et al. 2000 [ $[86,87]$, the scaling relation between the mass of the central MBH and the velocity dispersion of the stellar population of the spheroid bulge of galaxies $\left(M_{\mathrm{BH}}-\sigma_{\mathrm{sph}}\right)$, demonstrated the high likelihood of a co-evolution between the central MBH and its host galaxy and sparked a keen interest in the study of different scaling relations between the $M_{\mathrm{BH}}$ and the properties of its host. The tight correlation between the mass of the central $\mathrm{MBH}$ and the velocity dispersion of the galactic bulge suggests that there is mechanical feedback between the $\mathrm{MBH}$ and it host galaxy, which extends well beyond the sphere of gravitational influence of the $\mathrm{BH}$ and transcends the merger and/or accretion history of the MBH. While the origin of the coupling between the $\mathrm{MBH}$ and the host bulge velocity dispersion is not yet resolved, there is a multitude of analytic and numerical models that attempt to constrain the physical mechanism and reproduce the $M_{\mathrm{BH}}-\sigma_{\text {sph }}$ relation (e.g [ए05, ए06, ए07,

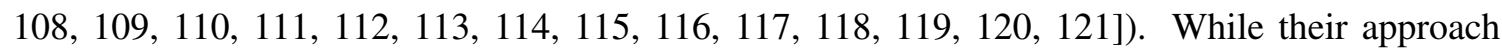
varies, all the above theoretical models rely on the presence of massive outflows or winds that are generated during the accretion episodes that are required to grow the central $\mathrm{MBH}$. The models can be divided into two broad categories depending on whether the interaction between the ouflow and the its host is "energy-driven" or "momentum-driven". The energy-driven models as suggested by Silk \& Rees (1998) [005] reproduce the scale of the relation, i.e. $M_{\mathrm{BH}} \sim \sigma_{\mathrm{sph}}{ }^{5}$ as suggested by more recent observational campaigns by McConnell \& Ma 2013, but significantly underestimate the normalization of the relation. On the other hand, momentum-driven solutions as suggested by 
King et al. (2003) [Ш]] predict a slope of $M_{\mathrm{BH}} \sim \sigma_{\mathrm{sph}}{ }^{4}$, but reproduce very well the $M_{\mathrm{BH}}$ for a given $\sigma_{\text {sph }}$.

Nevertheless, the observationally constrained $M_{\mathrm{BH}}-\sigma_{\text {sph }}$ relation itself is not well calibrated for low-mass galaxies, as the galactic samples employed to populate the relation suffer from luminosity bias and large uncertainties that are partially due to the precarious application of the $M_{\mathrm{BH}}-\sigma_{\text {sph }}$ relation in the low-mass regime (e.g. see discussion in [122, [23]). These issues result in an incompleteness of MBHs and host galaxies in the low-mass regime.

Another well-known scaling relation (and one which actually preceded the $M_{\mathrm{BH}}-\sigma_{\mathrm{sph}}$ ) is the

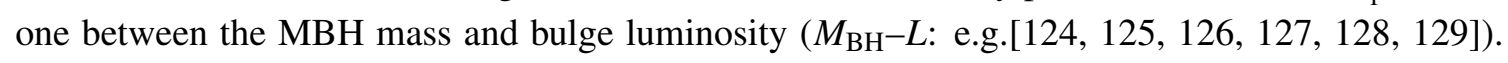
The tight scaling relation further underlines the co-relation between SMBH and galaxy growth, posing questions as to how this is regulated. Namely, is the feedback from the accreting SMBHs that promotes the growth of its host galaxy, or is the environment created by stellar activity that enables the growth of the SMBHs? The $M_{\mathrm{BH}}-L$ relation can be used to infer the mass of the central $\mathrm{MBH}$ and has the advantage that it does not require spectroscopy and can be applied to more distant galaxies. Studying low-mass galaxies using these two relations can reveal new IMBH candidates (e.g. [1530, [3] ]). However, the sampling of these two relations is biased towards higher luminosities/masses, as most galactic samples with directly measured SMBH masses are dominated by luminous bulges (e.g. [ए27, [32] ). Furthermore, there are large uncertainties regarding the shape or even the application of the $M_{\mathrm{BH}}-\sigma_{\text {sph }}$ relation in the low-mass regime (e.g see discussion in [122], [223, [133]).

In recent studies, Graham (2012) [ए34] and Graham \& Scott (2013) [ए28] revisited the $M_{\mathrm{BH}^{-}}$ $L$ including many more low-mass spheroids with directly measured SMBH masses. Their work dramatically revised the relation, demonstrating that it most likely follows a broken power-law relation, becoming steeper at low- and intermediate-mass spheroids (see also [ए35]. Using this improved scaling relation, [128] discovered 40 low luminosity AGN (LLAGN) that appear to have a mass of the central MBH that lies well within the IMBH range. However, Kormendy \& Ho (2013) [ए136] contest this result arguing that the steeper slope is the result of inclusion of pseudobulge galaxies, which are randomly offset towards lower masses and show little evidence of co-evolution between the central MBH and the host galaxy. On the other hand, Graham $(2012,2015)$ argues that a (steeper than linear) relation does exist for pseudobulges (and low-mass classical bulges), which indeed appear to be offset towards lower masses (for similar values of $L$ ) [एँ37, [138] and the "broken" $M_{\mathrm{BH}}-L$ holds. For a detailed discussion on this subject and also extensive reviews on SMBHs and their co-evolution with their host galaxies see Kormendy \& Ho (2013) [ए36] and Graham (2016) [एँ33].

Other MBH/host galaxy relations involve a correlation between the mass of a galaxy's central $\mathrm{BH}$ and its bulge concentration measured by the galactic Sérsic index (e.g. [1539, 1440, 144], 144], [43]). Furthermore, a correlation between the tightness of the spiral arms and the mass of the central MBH in disk galaxies was discovered by Seigar et al. (2008) [144]. This correlation was formulated as a relation between the $M_{\mathrm{BH}}$ and the spiral pitch angle (PA) which measures the tightness of the spiral arms [144, [45, [46]]. Each of these scaling relations may reveal possible candidates in the IMBH regime, however there are still large uncertainties in their estimations and the question of their validity in the low mass regime still remains. Nevertheless, in a recent publication [147]] four independent mass scaling relations and the fundamental plane of black hole 
activity (FP-BH: see next paragraph) were combined to measure the mass of the central MBH in seven LLAGN that had previously been reported to host IMBHs [ए28]. While the study did not confirm the previous estimations (the MBHs had a $\log M_{\mathrm{BH}} / M_{\odot} \approx 6.5$ on average), it demonstrated that all five (largely independent) methods produce consistent results in the low-mass regime. The study introduced this multiple-method/multi-wavelength approach as a go-to method for estimating central MBH masses when their spheres of gravitational influence cannot be spatially resolved. The combined techniques protect against outliers from any one relation and yield a more robust average prediction.

\subsection{IMBHs in AGN: The fundamental plane of black hole activity}

Another approach in the search for IMBHs draws on the well established relation between the X-ray emission, the radio emission and the mass of accreting BHs. These three quantities appear to be strongly correlated, forming what is known as the "fundamental plane of black hole activity" (FP-BH [148, [49]). During episodes of low-luminosity advection-dominated accretion (also known as a hard state), accretion disks are often accompanied by relativistic jets (e.g. [450, [5], [52]). During this state of accretion the accretion disk flow is interrupted at some distance from the central object, at which point the material flows radially towards the $\mathrm{BH}$. As a result of this new configuration, a "corona" of hot, optically-thin gas is created around the central object. When photons, from the (now truncated) accretion disk, traverse the "corona" they are Compton-upscattered to energies of tens to hundreds of keV. Furthermore the synchrotron mechanism in the relativistic jets produces emission primarily at the radio band (e.g. [453], [554, [55]). Therefore the combined emission of the accretion disk, corona and relativistic jets ranges from the radio to the hard X-ray band (see Done 2007 [156] and Gilfanov 2010 [157] for reviews of accreting compact objects). The entire broadband emission can also be dominated by the jet itself, particularly if accretion rates are higher $\left(\sim 0.01 \dot{M}_{\text {Edd }}\right.$, e.g. [1449]). An additional, less pronounced thermal component originating in the truncated accretion disk [158] may also be registered along with the primary non-thermal, coronal emission. While the details of the disk-jet mechanism are still far from resolved it is understood that the jet is linked directly to the accretion process. More importantly, it has been shown that the luminosities of the radio and X-ray emission are correlated and the disk-jet mechanism is independent of the BH mass [[5Y]. Based on these theoretical predictions, Merloni et al. (2003) [148] and Falcke (2004) [1449] probed a large sample of Galactic BHs and SMBHs and found a strong correlation between the radio luminosity $\left(\mathrm{L}_{\mathrm{r}}, 5 \mathrm{GHz}\right)$, the $\mathrm{X}$-ray luminosity $\left(\mathrm{L}_{\mathrm{x}}, 0.5-10 \mathrm{keV}\right)$, and the $\mathrm{BH}$ mass, known as the FP-BH.

The FP-BH can be used to infer the mass of accreting BHs during their hard-state. While this accretion state is more clearly defined in X-ray binaries (e.g. [1060, [16], [162]), the study of unobscured AGN shows that they can also be found in a similar accretion state (e.g. [163], [64, [65, [66, [6], [68, [69, [70]) and therefore the method can be employed to put constraints on the mass of central MBHs. The most likely candidates to host IMBHs are LLAGN in low-mass galaxies as they are expected to have undergone quiet merger histories and are, therefore, more likely to host lower-mass central BHs. While the X-ray and radio observations and the FP-BH can reveal IMBH candidates (e.g. [ए7], [47]), the scaling relation suffers from very high intrinsic scatter, therefore an estimation using solely the FP-BH would be very tentative. Nevertheless, as 
was demonstrated in Koliopanos et al. [147] it can provide a crucial, independent verification of estimations carried out using other methods.

\subsection{Extra-nuclear IMBHs}

\subsubsection{IMBHs and Globular clusters}

Under the right circumstances globular clusters (GCs) can become nurseries of IMBHs in the $10^{3} M_{\odot}$ range. Miller \& Hamilton (2002) [एT2] demonstrated that although consecutive three-body interactions may expel lighter BHs from GCs (e.g. [ए73], ए74, [75]), more massive BHs ( $250 M_{\odot}$ ) may sink towards the gravitational center of the cluster and after successive mergers with less massive BHs a $10^{3} M_{\odot}$ IMBH is generated. Simulations have also shown that, in sufficiently dense GCs, stellar collisions could result in a stellar "collision runaway" during which repeated collisions create enormously massive stars which collapse into IMBHs (e.g. [1776, [177, [38, [78]). While initial estimations of this process predicted IMBHs in the $10^{3} M_{\odot}$, further considerations of the uncertainties in the stellar evolution and mass loss of the merged systems showed that the resulting BHs could be as little as a few hundred $M_{\odot}$ mass [1779, 180, 187]. In the dense environment of GCs, IMBHs have a higher likelihood of capturing a companion. If the binding energy of the resulting binary is greater than the mean stellar kinetic energy in the cluster (a so called "hard binary"), repeated three body interactions will further harden the binary until accretion begins.

The presence of an IMBH - in the $10^{3} M_{\odot}$ range - in the center of a globular cluster is expected to effect its surrounding kinematics, in essence expanding the $M_{\mathrm{BH}}-\sigma_{\mathrm{sph}}$ relation to the lower mass regime. Estimations of the central velocity dispersion in GCs have revealed IMBH

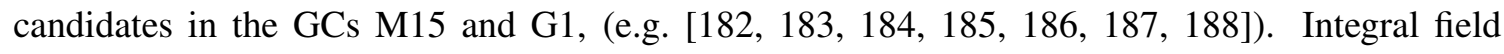
spectroscopy combined with surface brightness profiling and fitting to modeled slopes, predicted by N-body simulations, have also provided best fit estimations or upper limits for several more candidates (e.g. [189, [90, [1], [92]). Nevertheless, there is considerable controversy over the interpretation of these measurements, as these results can also be explained by an increased central concentration of stellar BHs or neutron stars (e.g [103], [194, [95, [96]). On the other hand, recent temporal studies of millisecond pulsars in globular clusters have indicated the presence of a central IMBH. Perera et al. (2017)[एव7] exploited 25 years of high precision observations of millisecond pulsar PSR B1820-30A, located in the globular cluster NGC 6624 to obtain rotational frequency time derivative measurements that can be interepreted as due to orbital motion around a central IMBH with $M>7500 M_{\odot}$. More recently Kiziltan et al. (2017) [ए98] probed the dynamics of the central region of GC 47 Tucanae by precisely measuring the location and acceleration of multiple millisecond X-ray pulsars found inside the cluster. Their results inferred the presence of a single, $2.3 \times 10^{3} M_{\odot}$ compact source ([प्8]], see section 4).

Evidence for the existence of IMBHs in GCs and measurements of their mass can be obtained if the IMBHs are accreting matter from a companion, or from surrounding gas. Namely, X-ray and/or radio emission may reveal the presence of an accreting $\mathrm{BH}$ and its mass can be measured using the FP-BH. With the exception of GC G1, no X-ray emission or radio emission has been detected in those GC IMBH candidates that have their presence inferred from kinematic indications. Radio observations of numerous GCs have so far only yielded upper limits on the mass of potential IMBHs in their center (assuming that they are there and they are accreting, e.g. [109, 200, 201, 202], 
but have also raised questions on their existence or whether they accrete any matter (e.g. Strader et al. 2010 [203] where no radio emission is detected in M15, M19, and M22, despite considerably deep observations or Wrobel et al. 2015 [204], who detect no radio emission in more than 300 extragalactic GCs in NGC 1032 using stacked observations). Deep X-ray observations of $\omega$ Centauri for which kinematic estimations suggest the existence of an IMBH [188, ए90] yielded no results, suggesting that either no IMBH is present or if it is, it is not accreting any mass [205]. The only GC with detections of both X-ray and radio emission is G1 in galaxy M31 [206, 207, 208], for which the FP-BH gives a consistent estimation with the $2 \times 10^{4} M_{\odot}$ reported by Gebhardt et al. [186, [87]. However, we must note that Miller-Jones et al. (2012) [209] re-observed G1 M31 using the VLA telescope and detected no radio emission down to a $3 \sigma$ upper limit of $4.7 \mathrm{muJy}$.

\subsubsection{IMBHs and ultraluminous $\mathrm{X}$-ray sources}

The search for IMBHs is not confined in the central MBHs of galaxies. Extra-nuclear IMBHs, produced inside dense globular clusters, can either sink towards to center of the cluster's gravitational potential well [37] or be gravitationally ejected from the cluster [210]. Extra-nuclear IMBHs may also be the remnants of past interactions of their host galaxies with other dwarf galaxies. If an IMBH finds itself in a binary system and is accreting material from its companion, its mass may be inferred by limits imposed on its accretion rate by physical laws, i.e. its Eddington limit. In accretion-powered sources the Eddington limit is imposed by the fact that when a certain threshold in the luminosity (corresponding to mass accretion rate) of the source is reached, the radiation pressure will exceed the ram pressure of the in-falling gas, thus hindering accretion and expelling the in-falling matter. In principle an accretion-powered source cannot be more luminous than its Eddington limit, which (as was shown by Eddington) scales linearly with the mass of the central object. Assuming spherical accretion of hydrogen, the Eddington limit for a stellar mass BH is $\sim 10^{39} \mathrm{erg} / \mathrm{s}$.

There are numerous off-nuclear, extragalactic X-ray sources with isotropic luminosities that exceed the Eddington limit for a stellar-mass BH, known as ultraluminous X-ray sources (ULXs) (see Kaaret 2017 [ [2] for a recent review). These sources were quickly considered as prime candidates for hosting IMBHs accreting at sub-Eddington rates in a similar fashion to standard stellar-mass XRBs (e.g. [2]2], [213], 214, [215]). While there is no known binary star evolution scenario that would result in an XRB with an IMBH accretor [2]6], an IMBH that has captured a companion could end up close enough with its companion to initiate mass accretion. This could either take place inside the cluster or the two companions may be driven closely, due to multiple few-body dynamical recoils as the binary exits it [217, 218, 219, 220]. However such a scenario would require most - if not all ULXs - to reside inside or close to globular clusters, which is not the case for the majority of ULXs. Furthermore, it has been demonstrated that most of the ULX population can be powered by stellar-mass BHs accreting at super-Eddington rates (e.g. [22], [222, [223, [224, [225]. The theoretical predictions are strongly backed by observations that reveal that the temporal and spectral characteristics of ULXs are markedly different from standard BHXRBs, suggesting that they indeed are in a different state of (super-Eddington) accretion, dubbed the ultraluminous state [226, [227, 228, [229]. The astounding discoveries of a pulsating ULX [230, [23], 232] demonstrated that ULXs can also be powered by neutron stars. A number of compelling theoretical studies have suggested that it is very likely that many (or most) ULXs may 
indeed be powered by neutron stars rather than BHs (e.g. [233], [234, 235]. Building on these theoretical predictions Koliopanos et al. (2017) [236] demonstrated in a recently published study that the spectral characteristics of a large fraction of ULXs are consistent with accretion onto highly magnetized neutron stars rather than BHs.

Nevertheless, there is a small group of hyper-luminous X-ray sources (HLXs) with luminosities exceeding $\sim 10^{41} \mathrm{erg} \mathrm{s}^{-1}$ that are hard to explain even when invoking super-Eddington accretion and/or beaming of the X-ray emission. HLXs are strong IMBH candidates, with a particular source, known as ESO 243-49 HLX-1 (henceforth HLX-1), considered as the best IMBH candidate found so far (see details in next section). Indeed HLX-1 and also M82 X-1 - the two brightest HLXs do not feature the spectral characteristics of ULXs (e.g. [237, [238]) and seem to follow the transitions from soft to hard state similar to sub-Eddington stellar-mass BH-XRBs (ESO 243-49 HLX-1: [68, [239, [240], or M82 X-1: [24]]).

\section{Notable candidates}

In this section, I present some notable IMBH candidates. The list is by far not comprehensive and only serves to present candidates with different origins, while also illustrating the results that can be achieved by different techniques.

\subsection{ULXs: HLX-1}

HLX-1 is probably the best IMBH candidate we know so far. Discovered serendipitously by Farrell et al. (2009) [238] in the 2XMM catalog [242], HLX-1 had an unabsorbed X-ray luminosity of $1.1 \times 10^{42} \mathrm{erg} \mathrm{s}^{-1}(0.2-10.0 \mathrm{keV})$, for a distance of $95 \mathrm{Mpc}$. Even if one assumes that the source is accreting at ten times the Eddington limit, this luminosity implies a minimum mass of $500 \mathrm{M}_{\odot}[238]$, making HLX-1 a prime IMBH candidate. This claim relies on the assumption that HLX-1 is indeed located in galaxy ESO 243-49, which lies at the aforementioned distance of $\mathrm{z}=0.023$. Indeed, in the years following the discovery of HLX-1 its association with ESO 243-49 has been confirmed by numerous studies. Using the accurate X-ray position derived from Chandra data [243], Soria et al. (2010) [244] located the source's optical counterpart, which shows an $\mathrm{H} \alpha$ emission line with a similar redshift to that of ESO 243-49, confirming it as its host galaxy [245], [246]. The exact origin of the $\mathrm{H} \alpha$ emission line is still unclear. If the line originates in an accretion disk [247], the disk must be observed almost face on, as the emission line is considerably narrow [246]. Wiersema et al. (2010) [248] suggested that the host star cluster or a photo-ionized/shockionized gas nebula very close to HLX-1 could be the source of the $\mathrm{H} \alpha$ emission, while Soria et al. 2010 [245] suggest a low density ionized nebula as the source. A more recent study of observations taken with the MUSE instrument on the VLT, combined with complementary multiwavelength data including X-Shooter, HST, Swift, Chandra and ATCA telescopes, revealed a strong variability of the emission line that was associated with the X-ray states of HLX-1. This finding confirms that the emission line originates close to the IMBH (negating the nebula or star cluster origin scenario) and validated the distance to HLX-1 [249].

HLX-1 - similarly to standard BH-XRBs - is also a source of occasional radio emission, which correlates with its transitions from hard (non thermal) to soft (thermal) spectral states [240]. Combining the X-ray and radio observations, the FP-BH allowed Webb et al. (2012) to place an 
upper limit of less than $10^{5} M_{\odot}$ for the mass of the $\mathrm{BH}$ in the system. This estimation was further supported by the estimation of a mass of $\sim 10^{4} M_{\odot}$ by Godet et al. (2012) [250], Davis et al. (2011) [D5]], and Straub et al. (2014) [D52] using accretion disk modeling. The X-ray flux of HLX-1 varies by a factor of 50 as the source transitions between hard and soft states [250]. This transition, along with the periodical appearance of jet emission (as the most likely origin of the radio emission), is a crucial feature that sets HLX-1 out as an outstanding candidate for an accreting IMBH. ULXs which are most likely super-Eddington accretors - do not transition between the strikingly distinct and characteristic "hard" and "soft" spectral states of standard BH-XRBs ( e.g. [253], 254, 228]]) and most frequently feature a characteristic exponential spectral cutoff at $\sim 10 \mathrm{keV}$, while the spectra of nominal BH-XRB extend to energies of often hundreds of $\mathrm{keV}$ during all spectral states. HLX1 , has a luminosity that is ten times higher than the brightest ULX and displays all the spectral and temporal characteristics of essentialy a scaled-up BH-XRBs, consistent with sub-Eddington accretion onto a very massive $(\gtrsim 500 M \odot[238]) \mathrm{BH}$.

The hyperluminous source [24] M82 X-1 has similar characteristics to HLX-1. However, it has not been observed as extensively as HLX-1, and recent works have shown that its spectrum can also be modeled assuming super-Eddington accretion onto a stellar mass $\mathrm{BH}$ [255]. Other notable sources in the same category involve the ULX NGC 2276-3C which exhibits powerful jet emission both in the X-ray and radio wavelengths, with an FP-BH derived mass of $\sim 5 \times 10^{4} M_{\odot}$ [256, 257] and the off nuclear X-ray source in NGC 5252 which radio and X-ray observations also place in the IMBH regime [ $[258]$. More recent optical integral-field observations of the surrounding kinematics of the source suggest that it may be an off-nuclear AGN, belonging to a small low-mass galaxy that is being accreted by the Seyfert galaxy NGC 5252 [250].

\subsection{Dwarf galaxies: NGC 4395}

NGC 4395 is an Sd type, bulgeless galaxy with a Seyfert 1 nucleus. The presence of an active $\mathrm{MBH}$ at the center of a bulgeless galaxy is exceptional in itself (see e.g. Ferrarese et al. 2000 [87], Merritt \& Ferrarese 2001 [260]), however the distinctness of NGC 4395 stems from the fact that it is one of the most likely candidates for a central, active IMBH. The presence of broadened optical and UV emission lines first revealed the AGN nature of NGC 4395 [26]]. Compact radio emission [262], suggestive of strong jet-like outflows [[263] and highly variable and non-thermal X-ray emission (e.g. [264, 265], 266, 267, 268]) solidified its AGN nature. More remarkably, the source's central stellar velocity dispersion was less than $\sim 30 \mathrm{~km} / \mathrm{s}$ [269], indicating a mass of the central MBH that is less than $10^{5} M_{\odot}$ based on the $M_{\mathrm{BH}}-\sigma_{\text {sph }}$ relation. This value is in agreement with estimations based on the broad profile of the $\mathrm{H} \beta$ line and from X-ray variability properties [269]. Further confirmation of a mass in the $10^{4}-10^{5} M_{\odot}$ range comes from reverberation mapping estimations (e.g. [05, [4]]) and from more recent kinematic estimations using integral field spectroscopy and fitting of the surface brightness of the nuclear star cluster [ $[0]$ ]. Despite the detection of both X-ray and radio emission from the core of NGC 4395, estimating the mass of the MBH using the FP-BH has been proven difficult due to the source's very high variability and the fact that the X-ray flux variability is only weakly coupled to the radio flux. Assuming the mass estimated by Peterson [05], the analysis of contemporaneous Swift (X-ray) and Very Large Array (radio) observations has hinted at the possibility of NGC 4395 lying at a steeper track of the FP-BH [270]. The presence of two distinct tracks in the FP-BH has been noted in stellar mass BH-XRBs, where 
state transitions occur at shorter intervals (e.g., [27], 272], 273], 274]). In addition to NGC 4395, some other strong central IMBH candidates, in dwarf galaxies are: NGC 404, for which dynamical estimations provide an upper limit of $1.5 \times 10^{5} M_{\odot}$ for the mass of its central $\mathrm{MBH}$ [275], an estimate that is further supported by X-ray and radio observations of its active nucleus [276, 275]; dwarf AGN POX 52, where multi-wavelength observations, including X-ray, radio, and $\mathrm{H} \beta$ optical emission suggest the presence of a $\sim 10^{5} M_{\odot} \mathrm{BH}$ in its center (e.g. [277]); LEDA 87000 (or RGG 118) whose central MBH lies in the intermediate mass regime, according to most $\mathrm{BH}$ mass - host bulge scaling relations [278], has the lowest BH mass measured in a dwarf galaxy and is also a low luminosity X-ray source [279]. Having had quiet merging and accretion histories, dwarf galaxies are strong candidates for hosting central IMBHs. A scenario that is further strengthened by recent extensive studies that indicate the presence of an IMBH population in dwarf galaxies up to a redshift of 2.4 [280, [28]].

\subsection{Globular clusters: 47 Tucanae}

The discovery of an IMBH candidate in the center of globular cluster 47 Tucanae is compelling not just for its own sake, but also for proposing a novel method to infer the presence and measure the mass of a gas-starved IMBH that is not electromagnetically visible. Kinematic estimations of the mass of a potential IMBH in the center of 47 Tuc yielded inconclusive results as N-body simulations revealed that velocity dispersion measurements alone could not distinguish between predictions that did or did not include a central IMBH [282, 283, [284]. Kizıltan et al. (2017)[108] took advantage of the presence of 19 millisecond pulsars located in the cluster, for which high resolution timing analysis was available. The authors measured the pulsar acceleration, detecting additional components (to the intrinsic source spin-up), which they demonstrated can be attributed to the presence of a central MBH in the cluster. Using this method they inferred the mass of the IMBH candidate to a value of $2300_{850}^{+1500} M_{\odot}$ and the mass of the globular cluster at $0.76 \times 10^{6} M_{\odot}$, in agreement with independent kinematic estimations [282, [284].

This novel method opens up the possibility for estimating the mass of other potential IMBHs in globular clusters for which electromagnetic detection is not feasible, but also to independently estimate the mass of IMBH candidates in GCs measured using more conventional methods. Nevertheless, there are caveats to the findings of Kiziltan et al.. Ridolfi et al. (2016)[285] and Freire et al. (2017)[[286] obtained 23 timing solutions for pulsars in 47 Tuc, including spin period derivatives, but also orbital period derivatives, and proper motion and jerk measurements. The authors argued that Kiziltan et al. had underestimated the distance to the cluster and by using an updated estimation for the distance, Freire et al. show that the all the accelerations of the pulsars in the clusters can be account for by a simple analytical model, without the need for a central IMBH.

\section{Conclusion: multi-wavelength future}

The extensive investigation by a large number of authors has yielded numerous tentative and several highly likely IMBH candidates, but a definitive detection of an IMBH has not happened yet. Nevertheless, the increasing number of plausible candidates discovered by multiple methods and at multiple wavelengths suggests that a population of IMBHs, very likely, exists but at this point its observational signatures lie at the threshold of our current observational limitations. 
Constraining this population and evaluating its characteristics is the next crucial step in the effort to understand how galaxies and globular clusters evolve. Determining the mass range in which central IMBHs lie (i.e. in the $10^{3}-10^{4} M_{\odot}$ or $\gtrsim 10^{5} M_{\odot}$ range) will largely decide on the formation path of SMBHs, as the different scenarios predict a starkly different shape of the $M_{\mathrm{BH}^{-}} \sigma_{\mathrm{sph}}$ distribution in the low-mass regime (e.g. Fig. 10 from Volonteri 2010 [40]). So far, galaxies with tentative measurements of their central MBH and velocity dispersion (e.g. [287, 288]) suggest the presence of a plume-like concentration of sources at the $10^{5} M_{\odot}$ mark (see also Volonteri 2010 [40] Mezcua 2017 [59] and references therein). While this preliminary, rough finding hints towards the dominance of the massive seeds scenarios, it is equally plausible that the lower mass IMBHs remain below our observational capabilities as their AGN are luminosity and distance limited and the $M_{\mathrm{BH}}-\sigma_{\text {sph }}$ distribution is populated primarily by MBHs that have ended up above the IMBH regime, via accretion and mergers. It becomes apparent from the multitude of studies, briefly reviewed above, that multi-wavelength observations hold the key to constraining the missing population of IMBHs. As we enter the era of multi-messenger astronomy and push the threshold of our observational capabilities, the detection and classification of IMBHs promises to be one of the big discoveries of the next decade.

\section{DISCUSSION}

DANIELE FARGION: I would like to also point out the case of M82 X-1 as well known candidate for an IMBH [Author's note: M82 X-1 had not been discussed during the presentation ].

FILIPPOS KOLIOPANOS: Indeed, M82 X-1 is one of the two best candidate IMBH hyperluminous sources. The source shows similar characteristics as HLX-1 and appears to behave like a sub-Eddington BH-XRB in the intermediate mass range. The reason the source was not mentioned in the oral presentation and is not discussed as extensively as HLX-1 in the review, is that M82 $\mathrm{X}-1$ has not been monitored as extensively as HLX-1 which has been systematically observed at all wavelength, and also the fact that more recent estimations point out that M82 X-1 can be plausibly described as a super-Eddington source.

WOLFGANG KUNDT: How do you explain the problem of the naked singularity? [Author's note: Prof. Kundt has argued for a long time against the existence of BHs, and has also criticized Roger Penrose's cosmic censorship hypothesis. See e.g. Astrophysics Without Black Holes, and Without Extragalactic Gamma-Ray Bursts

https://ojs.cvut.cz/ojs/index.php/APP/article/view/APP.2014.01.0027 ]

FILIPPOS KOLIOPANOS: As I am not a theoretical physicist or mathematician, I cannot comment - at an expert level - on the potential problems of the cosmic censorship hypothesis. However, as an observer I am satisfied by the currently available observational evidence in favor of the presence of SMBHs - and more importantly their interpretation in the context of general relativity. By "evidence" I am mostly referring to three observational milestones in the SMBH hypothesis. The detection of highly broadened, $6.4 \mathrm{keV}$ iron $\mathrm{K} \alpha$ lines in AGN (starting with the galaxy MCG-6-3015 by Tanaka et al. 1995), that are consistent with gravitational redshift by compact objects with a 
mass that exceeds $10^{6} M_{\odot}$. The independent estimations from megamaser emission (e.g. Miyoshi et al. 1995) displaying clear Keplerian rotation curves that require a mass of the order of $\sim 10^{7} M_{\odot}$ and finally the dynamical evidence for the SMBH in the center of the Milky Way (e.g. Schödel et al. 2002).

\section{Acknowledgments}

The author would like to than Mar Mezcua for refereeing and significantly improving this review and Franco Giovannelli for giving me the opportunity to present this work and be part of the XII Multifrequency Behaviour of High Energy Cosmic Sources Workshop .

\section{References}

[1] J. Droste, The field of a single centre in einstein's theory of gravitation, and the motion of a particle in that field, Ned. Acad. Wet., SA 19 (1917) 197.

[2] Hilbert, D. 1917, Nachr. Ges. Wiss. Gottingen, Math. Phys. K1., 53

[3] Weyl, H. 1917, Ann. Phys. (Leipzig) 54, 117

[4] Landau, L. D. 1932, Phys. Z. Sowjetunion, 1, 285

[5] Chandrasekhar, S. 1932, Zeitschrift für Astrophysik, Vol. 5, p.32

[6] S. Chandrasekhar, The highly collapsed configurations of a stellar mass (Second paper), MNRAS 95 (Jan., 1935) 207-225.

[7] B. L. Webster and P. Murdin, Cygnus X-1-a Spectroscopic Binary with a Heavy Companion ?, Nature 235 (Jan., 1972) 37-38.

[8] R. A. Remillard and J. E. McClintock, X-Ray Properties of Black-Hole Binaries, ARA\&A 44 (Sept., 2006) 49-92, [astro-ph/0606352].

[9] J. Casares and P. G. Jonker, Mass Measurements of Stellar and Intermediate-Mass Black Holes, Space $\sim$ Sc1. Rev. 183 (Sept., 2014) 223-252, [1311.5118].

[10] B. P. Abbott, R. Abbott, T. D. Abbott, M. R. Abernathy, F. Acernese, K. Ackley et al., GW151226: Observation of Gravitational Waves from a 22-Solar-Mass Binary Black Hole Coalescence, Physical Review Letters 116 (June, 2016) 241103, [11606.04855].

[11] B. P. Abbott, R. Abbott, T. D. Abbott, F. Acernese, K. Ackley, C. Adams et al., GW170104: Observation of a 50-Solar-Mass Binary Black Hole Coalescence at Redshift 0.2, Physical Review Letters 118 (June, 2017) 221101, [11706.01812].

[12] B. P. Abbott, R. Abbott, T. D. Abbott, F. Acernese, K. Ackley, C. Adams et al., GW170814: A Three-Detector Observation of Gravitational Waves from a Binary Black Hole Coalescence, Physical Review Letters 119 (Oct., 2017) 14110], [1709.09660].

[13] R. H. Sanders and T. Lowinger, The Distribution of Mass in the Galactic Nucleus, AJ77 (May, 1972) 292.

[14] M. J. Rees, Black Hole Models for Active Galactic Nuclei, ARA\&A 22 (1984) 471-506.

[15] Y. Tanaka, K. Nandra, A. C. Fabian, H. Inoue, C. Otani, T. Dotani et al.,

Gravitationally redshifted emission implying an accretion disk and massive black hole in the active galaxy MCG-6-30-15 Nature 375 (June, 1995) 659-661. 
[16] M. Miyoshi, J. Moran, J. Herrnstein, L. Greenhill, N. Nakai, P. Diamond et al., Evidence for a black hole from high rotation velocities in a sub-parsec region of NGC4258, Nature B73 (Jan., 1995) 127-129.

[17] R. Schödel, T. Ott, R. Genzel, R. Hofmann, M. Lehnert, A. Eckart et al., A star in a 15.2-year orbit around the supermassive black hole at the centre of the Milky Way, Nature 419 (Oct., 2002) 694-696, [astro-ph/0210426].

[18] Z. Haiman, A. A. Thoul and A. Loeb, Cosmological Formation of Low-Mass Objects, ApJ 464] [June, 1996) 523, astro-ph/9507111].

[19] N. Yoshida, T. Abel, L. Hernquist and N. Sugiyama, Simulations of Early Structure Formation: Primordial Gas Clouds, ApJ 592(Aug., 2003) 645-663, [astro-ph/0301645].

[20] V. Bromm, P. S. Coppi and R. B. Larson, Forming the First Stars in the Universe: The Fragmentation of Primordial Gas, ApJ 527 (Dec., 1999) [L5-L8, [astro-ph/9910224].

[21] V. Bromm, P. S. Coppi and R. B. Larson,

The Formation of the First Stars. I. The Primordial Star-forming Cloud, ApJ 564 (Jan., 2002) 23-51, [astro-ph/0102503].

[22] F. Nakamura and M. Umemura, On the Initial Mass Function of Population III Stars, ApJ 548 (Feb., 2001) 19-32, astro-ph/0010464].

[23] T. Abel, G. L. Bryan and M. L. Norman, The Formation of the First Star in the Universe, Science 295(Jan., 2002) 93-98, [astro-ph/0112088].

[24] K. Omukai, Protostellar Collapse with Various Metallicities, ApJ 534(May, 2000) 809-824, [astro-ph/0003212].

[25] K. Omukai and F. Palla, Formation of the First Stars by Accretion, Ap J589 (June, 2003) 677-687, [astro-ph/0302345].

[26] B. W. O'Shea and M. L. Norman, Population III Star Formation in a $\Lambda$ CDM Universe. I. The Effect of Formation Redshift and Environment on Protostellar ApJ 654 (Jan., 2007) 66-92, [astro-ph/0607013].

[27] P. Madau and M. J. Rees, Massive Black Holes as Population III Remnants, ApJ 551(Apr., 2001) [L27-L30, astro-ph/0101223].

[28] M. Ricotti and J. P. Ostriker,

X-ray pre-ionization powered by accretion on the first black holes - I. A model for the WMAP polarization measurement MNRAS 352(Aug., 2004) 547-562, astro-ph/0311003].

[29] J. C. Wheeler and V. Johnson, Stellar-mass Black Holes in Young Galaxies, ApJ 738(Sept., 2011) [163], [1107.3165].

[30] C.-E. Rydberg, E. Zackrisson, P. Lundqvist and P. Scott, Detection of isolated Population III stars with the James Webb Space Telescope, MNRAS 429 [Mar., 2013) 3658-3664, [1206.0007].

[31] B. McKernan, K. E. S. Ford, W. Lyra and H. B. Perets, Intermediate mass black holes in AGN discs - I. Production and growth, MNRAS 425 (Sept., 2012) 460-469, [1206.2309]. 
[32] B. McKernan, K. E. S. Ford, B. Kocsis, W. Lyra and L. M. Winter, Intermediate-mass black holes in AGN discs - II. Model predictions and observational constraints, MNRAS 441 (June, 2014) 900-909, [1403.6433].

[33] J. M. Bellovary, M.-M. Mac Low, B. McKernan and K. E. S. Ford, Migration Traps in Disks around Supermassive Black Holes, ApJ 819 (Mar., 2016) L17, [1511.00005].

[34] Y. Taniguchi, Y. Shioya, T. G. Tsuru and S. Ikeuchi, Formation of Intermediate-Mass Black Holes in Circumnuclear Regions of Galaxies, PASJ 52 (June, 2000) 533-537, [astro-ph/0002389].

[35] M. C. Miller and D. P. Hamilton, Production of intermediate-mass black holes in globular clusters, MNRAS 330 (Feb., 2002) 232-240, [astro-ph/0106188].

[36] S. F. Portegies Zwart and S. L. W. McMillan, The Runaway Growth of Intermediate-Mass Black Holes in Dense Star Clusters, ApJ 576 (Sept., 2002) 899-907, [astro-ph/0201055].

[37] S. F. Portegies Zwart, H. Baumgardt, P. Hut, J. Makino and S. L. W. McMillan, Formation of massive black holes through runaway collisions in dense young star clusters, Nature 228(Apr., 2004) 724-726, [astro-ph/0402622].

[38] M. A. Gürkan, M. Freitag and F. A. Rasio, Formation of Massive Black Holes in Dense Star Clusters. I. Mass Segregation and Core Collapse, ApJ 604(Apr., 2004) 632-652, [astro-ph/0308449].

[39] M. Freitag, M. A. Gürkan and F. A. Rasio, Runaway collisions in young star clusters - II. Numerical results, MNRAS 368 (May, 2006) [41-16], [astro-ph/0503130].

[40] M. Volonteri, Formation of supermassive black holes, The Astronomy and Astrophysics Review 18 (2010) 279-315.

[41] T. Abel, G. L. Bryan and M. L. Norman, The formation of the first star in the universe, science 295 (2002) 93-98.

[42] V. Bromm, P. S. Coppi and R. B. Larson, The formation of the first stars. i. the primordial star-forming cloud, The Astrophysical Journal 564 (2002) 23.

[43] M. J. Turk, T. Abel and B. O'shea, The formation of population iii binaries from cosmological initial conditions, Science 325 (2009) 601-605.

[44] T. Tanaka and Z. Haiman, The assembly of supermassive black holes at high redshifts, The Astrophysical Journal 696 (2009) 1798.

[45] M. Volonteri, P. Madau and F. Haardt,

The Formation of Galaxy Stellar Cores by the Hierarchical Merging of Supermassive Black Holes, ApJ 593 (Aug., 2003) 661-666, [astro-ph/0304389].

[46] T. Ryu, T. L. Tanaka, R. Perna and Z. Haiman, Intermediate-mass black holes from Population III remnants in the first galactic nuclei, MNRAS 46I (Aug., 2016) 4122-4134, [1603.08513].

[47] D. Whalen, T. Abel and M. L. Norman, Radiation Hydrodynamic Evolution of Primordial H II Regions, ApJ 610 (July, 2004) 14-22. [astro-ph/0310283]. 
[48] D. J. Whalen and C. L. Fryer,

The Formation of Supermassive Black Holes from Low-mass Pop III Seeds, ApJ 756 (Sept., 2012) LIV, [1112.4598].

[49] B. Agarwal, S. Khochfar, J. L. Johnson, E. Neistein, C. Dalla Vecchia and M. Livio, Ubiquitous seeding of supermassive black holes by direct collapse, MNRAS 425 (Oct., 2012) [2854-2877, [1205.6464]].

[50] T. E. Woods, A. Heger, D. J. Whalen, L. Haemmerlé and R. S. Klessen, On the Maximum Mass of Accreting Primordial Supermassive Stars, ApJ 842 (June, 2017) L6, [1703.07480].

[51] S. P. Oh, Reionization by Hard Photons. I. X-Rays from the First Star Clusters, ApJ 553 (June, 2001) 499-512, [astro-ph/0005262].

[52] M. Ricotti, N. Y. Gnedin and J. M. Shull, Feedback from Galaxy Formation: Production and Photodissociation of Primordial $\mathrm{H}_{2}$, Ap $\mathbf{5 6 0}$ Oct., 2001) 580-59], [astro-ph/0012335].

[53] S. C. O. Glover and P. W. J. L. Brand, Radiative feedback from an early X-ray background, MNRAS B40 (Mar., 2003) 210-226, [astro-ph/0205308].

[54] M. E. Machacek, G. L. Bryan and T. Abel, Effects of a soft X-ray background on structure formation at high redshift, MNRAS 338 (Jan., 2003) 273-286, [astro-ph/0209326].

[55] M. Kuhlen and P. Madau, The first miniquasar, MNRAS 363 (Nov., 2005) 1069-1082 [astro-ph/0506712].

[56] S. Zaroubi, R. M. Thomas, N. Sugiyama and J. Silk, Heating of the intergalactic medium by primordial miniquasars, MNRAS 375 (Mar., 2007) [1269-1279, [astro-ph/0609151].

[57] E. Ripamonti, M. Mapelli and S. Zaroubi, Radiation from early black holes - I. Effects on the neutral intergalactic medium, MNRAS 387 [June, 2008) 158-172, [0802.1857].

[58] R. M. Thomas and S. Zaroubi, Time-evolution of ionization and heating around first stars and miniqsos, MNRAS 384 (Mar., 2008) 1080-1096, [0709.1657].

[59] M. Mezcua, Observational evidence for intermediate-mass black holes, nnternationaL Journa of Modern Physics D 26 (2017) 1730021, [1705.09667].

[60] M. Volonteri, G. Lodato and P. Natarajan, The evolution of massive black hole seeds, MNRAS 383 [Jan., 2008) 1079-1088, [0709.0520].

[61] H. Shirakata, T. Kawaguchi, T. Okamoto, R. Makiya, T. Ishiyama, Y. Matsuoka et al., Theoretical re-evaluations of the black hole mass-bulge mass relation - I. Effect of seed black hole mass, MNRAS 461 (Oct., 2016) 4389-4394, [1604.05317].

[62] Y. B. Zel'dovich and I. D. Novikov,

The Hypothesis of Cores Retarded during Expansion and the Hot Cosmological Model, Soviet Ast. 10 (Feb., 1967) 602.

[63] S. W. Hawking, I. G. Moss and J. M. Stewart, Bubble collisions in the very early universe, Phys. Rev. D 26 (Nov., 1982) 2681-2693. 
[64] K. Jedamzik and J. C. Niemeyer, Primordial black hole formation during first-order phase transitions, Bhys. Rev. D 59 (June, 1999) [124014, [astro-ph/9901293].

[65] S. G. Rubin, M. Y. Khlopov and A. S. Sakharov, Primordial Black Holes from Non-Equilibrium Second Order Phase Transition, ArXiv High Energy Physics - Phenomenology e-prints (May, 2000), [hep-ph/0005271].

[66] S. G. Rubin, A. S. Sakharov and M. Y. Khlopov, The Formation of Primary Galactic Nuclei during Phase Transitions in the Early Universe, Soviet Journal of Experimental and Theoretical Physics 92 (June, 2001) 921-929, [hep-ph/0106187].

[67] M. Y. Khlopov, S. G. Rubin and A. S. Sakharov, Primordial structure of massive black hole clusters, Astroparticle Physics 23 (Mar., 2005) 265-277, [astro-ph/0401532].

[68] O. Godet, D. Barret, N. A. Webb, S. A. Farrell and N. Gehrels, First Evidence for Spectral State Transitions in the ESO 243-49 Hyperluminous X-Ray Source HLX-1, ApJ 705 (Nov., 2009) LI09-LI12, [0909.4458].

[69] A. M. Ghez, S. Salim, S. D. Hornstein, A. Tanner, J. R. Lu, M. Morris et al., Stellar Orbits around the Galactic Center Black Hole, ApJ 620 (Feb., 2005) 744-757, [astro-ph/0306130].

[70] R. Genzel, F. Eisenhauer and S. Gillessen, The Galactic Center massive black hole and nuclear star cluster, Reviews of Modern Physics 82 (Oct., 2010) 3121-3195, [1006.0064]].

[71] L. Meyer, A. M. Ghez, R. Schödel, S. Yelda, A. Boehle, J. R. Lu et al., The Shortest-Known-Period Star Orbiting Our Galaxy's Supermassive Black Hole, Science 338 (Oct., 2012) 84, [1210.1294].

[72] F. Macchetto, A. Marconi, D. J. Axon, A. Capetti, W. Sparks and P. Crane, The supermassive black hole of $\mathrm{m} 87$ and the kinematics of its associated gaseous disk, The Astrophysical Journal 489 (1997) 579.

[73] R. P. van der Marel and F. C. van den Bosch, Evidence for a 3× 108 mâŸL' black hole in ngc 7052 from hubble space telescope observations of the nuclear gas disk, The Astronomical Journal 116 (1998) 2220.

[74] G. A. V. Kleijn, R. P. van der Marel, C. M. Carollo and P. T. de Zeeuw, The black hole in ic 1459 from hubble space telescope observations of the ionized gas disk, The Astronomical Journal 120 (2000) 1221.

[75] A. J. Barth, M. Sarzi, H.-W. Rix, L. C. Ho, A. V. Filippenko and W. L. Sargent, Evidence for a supermassive black hole in the s0 galaxy ngc 3245, The Astrophysical Journal 555 (2001) 685.

[76] M. Sarzi, H.-W. Rix, J. C. Shields, G. Rudnick, L. C. Ho, D. H. McIntosh et al., Supermassive black holes in bulges, The Astrophysical Journal 550 (2001) 65.

[77] N. Neumayer, M. Cappellari, J. Reunanen, H.-W. Rix, P. Van Der Werf, P. De Zeeuw et al., The central parsecs of centaurus a: high-excitation gas, a molecular disk, and the mass of the black hole, The Astrophysical Journal 671 (2007) 1329.

[78] P. Peebles, Star distribution near a collapsed object, The Astrophysical Journal 178 (1972) 371-376.

[79] J. Frank and M. J. Rees, Effects of massive central black holes on dense stellar systems, Monthly Notices of the Royal Astronomical Society 176 (1976) 633-647. 
[80] M. Den Brok, A. C. Seth, A. J. Barth, D. J. Carson, N. Neumayer, M. Cappellari et al., Measuring the mass of the central black hole in the bulgeless galaxy ngc 4395 from gas dynamical modeling, The Astrophysical Journal 809 (2015) 101.

[81] A. C. Seth, M. Cappellari, N. Neumayer, N. Caldwell, N. Bastian, K. Olsen et al., The ngc 404 nucleus: star cluster and possible intermediate-mass black hole, The Astrophysical Journal $\mathbf{7 1 4}$ (2010) 713 .

[82] M. Valluri, L. Ferrarese, D. Merritt and C. L. Joseph, The low end of the supermassive black hole mass function: constraining the mass of a nuclear black hole in ngc 205 via stellar kinematics, The Astrophysical Journal 628 (2005) 137.

[83] V. Lora, F. Sánchez-Salcedo, A. Raga and A. Esquivel, An upper limit on the mass of the black hole in ursa minor dwarf galaxy, The Astrophysical Journal Letters 699 (2009) L113.

[84] J. R. Jardel and K. Gebhardt, The dark matter density profile of the fornax dwarf, The Astrophysical Journal 746 (2012) 89.

[85] B. M. Peterson, Measuring the masses of supermassive black holes, Space Science Reviews $\mathbf{1 8 3}$ (2014) 253-275.

[86] L. Ferrarese and D. Merritt, A Fundamental Relation between Supermassive Black Holes and Their Host Galaxies, ApJ539 Aug., 2000) L9-L12, astro-ph/0006053.

[87] K. Gebhardt, R. Bender, G. Bower, A. Dressler, S. M. Faber, A. V. Filippenko et al., A Relationship between Nuclear Black Hole Mass and Galaxy Velocity Dispersion, ApJ539(Aug., 2000) L13-L16, [astro-ph/0006289].

[88] C. A. Onken, L. Ferrarese, D. Merritt, B. M. Peterson, R. W. Pogge, M. Vestergaard et al., Supermassive black holes in active galactic nuclei. ii. calibration of the black hole mass-velocity dispersion relationship for active galactic nuclei, The Astrophysical Journal 615 (2004) 645.

[89] S. Collin, T. Kawaguchi, B. M. Peterson and M. Vestergaard, Systematic effects in measurement of black hole masses by emission-line reverberation of active galactic nuclei: Eddington ratio and inclination, Astronomy \& Astrophysics 456 (2006) 75-90.

[90] J. E. Greene, C. Y. Peng, M. Kim, C.-Y. Kuo, J. A. Braatz, C. V. Impellizzeri et al., Precise black hole masses from megamaser disks: black hole-bulge relations at low mass, The Astrophysical Journal 721 (2010) 26.

[91] A. W. Graham, C. A. Onken, E. Athanassoula and F. Combes, An expanded m bh- $\sigma$ diagram, and a $\underline{\text { new calibration of active galactic nuclei masses, }}$ Monthly Notices of the Royal Astronomical Society 412 (2011) 2211-2228.

[92] C. Grier, P. Martini, L. Watson, B. Peterson, M. Bentz, K. Dasyra et al., Stellar velocity dispersion measurements in high-luminosity quasar hosts and implications for the agn black hole mass scale, The Astrophysical Journal 773 (2013) 90.

[93] J. E. Mejía-Restrepo, P. Lira, H. Netzer, B. Trakhtenbrot and D. M. Capellupo, The effect of nuclear gas distribution on the mass determination of supermassive black holes, Nature Astronomy 2 (Jan., 2018) 63-68, [1709.05345].

[94] H. Edri, S. E. Rafter, D. Chelouche, S. Kaspi and E. Behar, Broadband photometric reverberation mapping of ngc 4395, The Astrophysical Journal 756 (2012) 73. 
[95] B. M. Peterson, M. C. Bentz, L.-B. Desroches, A. V. Filippenko, L. C. Ho, S. Kaspi et al., Multiwavelength monitoring of the dwarf seyfert 1 galaxy ngc 4395. i. a reverberation-based measurement of the black hole mass, The Astrophysical Journal 632 (2005) 799.

[96] S. Kaspi, P. S. Smith, H. Netzer, D. Maoz, B. T. Jannuzi and U. Giveon, Reverberation measurements for 17 quasars and the size-mass-luminosity relations in active galactic nuclei, The Astrophysical Journal 533 (2000) 631.

[97] R. J. McLure and J. Dunlop, The black hole masses of seyfert galaxies and quasars, Monthly Notices of the Royal Astronomical Society 327 (2001) 199-207.

[98] R. J. McLure and M. J. Jarvis, Measuring the black hole masses of high-redshift quasars, Monthly Notices of the Royal Astronomical Society 337 (2002) 109-116.

[99] M. Vestergaard, Determining central black hole masses in distant active galaxies, The Astrophysical Journal 571 (2002) 733.

[100] M. Vestergaard and B. M. Peterson, Determining central black hole masses in distant active galaxies and quasars. ii. improved optical and uv scaling relationships, The Astrophysical Journal 641 (2006) 689.

[101] J.-G. Wang, X.-B. Dong, T.-G. Wang, L. C. Ho, W. Yuan, H. Wang et al., Estimating black hole masses in active galactic nuclei using the $\mathrm{mg}$ ii $\lambda 2800$ emission line, The Astrophysical Journal 707 (2009) 1334.

[102] J. A. Baldwin, M. M. Phillips and R. Terlevich, Classification parameters for the emission-line spectra of extragalactic objects, PASP93 (Feb. 1981) 5-19.

[103] J. E. Greene and L. C. Ho, Active Galactic Nuclei with Candidate Intermediate-Mass Black Holes, ApJ610(Aug., 2004) 722-736, [astro-ph/0404110].

[104] A. E. Reines, J. E. Greene and M. Geha, Dwarf Galaxies with Optical Signatures of Active Massive Black Holes, ApJ775 (Oct., 2013)116, [1308.0328].

[105] J. Silk and M. J. Rees, Quasars and galaxy formation, arXiv preprint astro-ph/9801013 (1998) .

[106] M. G. Haehnelt, P. Natarajan and M. J. Rees, High-redshift galaxies, their active nuclei and central black holes, Monthly Notices of the Royal Astronomical Society 300 (1998) 817-827.

[107] R. D. Blandford, Origin and Evolution of Massive Black Holes in Galactic Nuclei, in Galaxy Dynamics - A Rutgers Symposium (D. R. Merritt, M. Valluri and J. A. Sellwood, eds.), vol. 182 of Astronomical Society of the Pacific Conference Series, Aug., 1999, astro-ph/9906025.

[108] A. Fabian, The obscured growth of massive black holes, Monthly Notices of the Royal Astronomical Society 308 (1999) L39-L43.

[109] M. G. Haehnelt and G. Kauffmann, The correlation between black hole mass and bulge velocity dispersion in hierarchical galaxy formation models, Monthly Notices of the Royal Astronomical Society 318 (2000) L35-L38.

[110] A. Benson, R. Bower, C. Frenk, C. G. Lacey, C. Baugh and S. Cole, What shapes the luminosity function of galaxies?, The Astrophysical Journal 599 (2003) 38.

[111] A. King, Black holes, galaxy formation, and the mbh- $\sigma$ relation, The Astrophysical Journal Letters 596 (2003) L27. 
[112] J. S. B. Wyithe and A. Loeb, Self-regulated growth of supermassive black holes in galaxies as the origin of the optical and x-ray luminosity functions of quasars, The Astrophysical Journal $\mathbf{5 9 5}$ (2003) 614.

[113] G. L. Granato, G. De Zotti, L. Silva, A. Bressan and L. Danese, A physical model for the coevolution of qsos and their spheroidal hosts, The Astrophysical Journal 600 (2004) 580.

[114] T. Di Matteo, V. Springel and L. Hernquist, Energy input from quasars regulates the growth and activity of black holes and their host galaxies, Nature 433 (2005) 604-607.

[115] V. Springel, S. D. White, A. Jenkins, C. S. Frenk, N. Yoshida, L. Gao et al., Simulations of the formation, evolution and clustering of galaxies and quasars, nature 435 (2005) 629-636.

[116] P. F. Hopkins, L. Hernquist, P. Martini, T. J. Cox, B. Robertson, T. Di Matteo et al., A physical model for the origin of quasar lifetimes, The Astrophysical Journal Letters 625 (2005) L71.

[117] D. Sijacki, V. Springel, T. Di Matteo and L. Hernquist, A unified model for agn feedback in cosmological simulations of structure formation, Monthly Notices of the Royal Astronomical Society 380 (2007) 877-900.

[118] R. S. Somerville, P. F. Hopkins, T. J. Cox, B. E. Robertson and L. Hernquist, A semi-analytic model for the co-evolution of galaxies, black holes and active galactic nuclei, Monthly Notices of the Royal Astronomical Society 391 (2008) 481-506.

[119] C. Booth and J. Schaye, Cosmological simulations of the growth of supermassive black holes and feedback from active galactic nuclei: method and tests, Monthly Notices of the Royal Astronomical Society 398 (2009) 53-74.

[120] K. Zubovas and A. R. King, The $\mathrm{m}-\sigma$ relation in different environments, Monthly Notices of the Royal Astronomical Society 426 (2012) 2751-2757.

[121] T. Costa, D. Sijacki and M. G. Haehnelt, Feedback from active galactic nuclei: energy-versus momentum-driving, Monthly Notices of the Royal Astronomical Society 444 (2014) 2355-2376.

[122] J. E. Greene and L. C. Ho, The Mass Function of Active Black Holes in the Local Universe, ApJ 667 Sept., 2007) 131-148, [0705.0020].

[123] E. C. Moran, K. Shahinyan, H. R. Sugarman, D. O. Vélez and M. Eracleous, Black Holes At the Centers of Nearby Dwarf Galaxies, AJ 148 (Dec., 2014) 136, [11408.4451].

[124] A. Dressler, Observational Evidence for Supermassive Black Holes, in Active Galactic Nuclei (D. E. Osterbrock and J. S. Miller, eds.), vol. 134 of IAU Symposium, p. 217, 1989.

[125] J. Kormendy, ed., in "The nearest active galaxies", edited by Beckman,J. and Colina, L. and Netzer, H., 1993.

[126] J. Magorrian, S. Tremaine, D. Richstone, R. Bender, G. Bower, A. Dressler et al., The Demography of Massive Dark Objects in Galaxy Centers, AJ115 (June, 1998) 2285-2305, [astro-ph/9708072].

[127] A. Marconi and L. K. Hunt, The Relation between Black Hole Mass, Bulge Mass, and Near-Infrared Luminosity, ApJ 589(May, 2003) L21-L24, [astro-ph/0304274].

[128] A. W. Graham and N. Scott,

The $\mathrm{M}_{B H}-\mathrm{L}_{\text {spheroid }}$ Relation at High and Low Masses, the Quadratic Growth of Black Holes, and Intermediate-mass Bla ApJ 764(Feb., 2013) 151, [1211.3199]. 
[129] G. A. D. Savorgnan, A. W. Graham, A. Marconi and E. Sani, Supermassive Black Holes and Their Host Spheroids. II. The Red and Blue Sequence in the $\mathrm{M}_{B H}-\mathrm{M}_{*, s p h}$ Diagram, ApJ817 (Jan., 2016) 21], [1511.07437].

[130] A. J. Barth, J. E. Greene and L. C. Ho, Low-Mass Seyfert 2 Galaxies in the Sloan Digital Sky Survey, AJ136 (Sept., 2008) 1179-1200, [0807.3316].

[131] X.-B. Dong, L. C. Ho, W. Yuan, T.-G. Wang, X. Fan, H. Zhou et al., A Uniformly Selected Sample of Low-mass Black Holes in Seyfert 1 Galaxies, ApJ 755 (Aug.) 2012) 167, [1206.3843].

[132] A. Graham, A Significantly Reduced Scatter In The $\mathrm{M}_{B h^{-}}-\sigma$ Relation, in American Astronomical Society Meeting Abstracts, vol. 39 of Bulletin of the American Astronomical Society, p. 759, Dec., 2007.

[133] A. W. Graham, Galaxy bulges and their massive black holes: a review, in Galactic Bulges, pp. 263-313. Springer, 2016.

[134] A. W. Graham, Breaking the Law: The $\mathrm{M}_{b h}-\mathrm{M}_{\text {spheroid }}$ Relations for Core-Sérsic and Sérsic Galaxies, ApJ 746 [Feb., 2012) 113], [1202.1878].

[135] A. W. Graham and N. Scott, The (Black Hole)-bulge Mass Scaling Relation at Low Masses, ApJ 798 [Jan., 2015) 54, [1412.3091].

[136] J. Kormendy and L. C. Ho, Coevolution (or not) of supermassive black holes and host galaxies, Annual Review of Astronomy and Astrophysics 51 (2013) 511-653.

[137] A. W. Graham, Populating the galaxy velocity dispersion: Supermassive black hole mass diagram, a catalogue of ( $\mathrm{m}$ bh, $\sigma$ ) values, Publications of the Astronomical Society of Australia 25 (2008) $167-175$.

[138] J. Hu, The black hole mass-stellar velocity dispersion correlation: bulges versus pseudo-bulges, Monthly Notices of the Royal Astronomical Society 386 (2008) 2242-2252.

[139] A. W. Graham, P. Erwin, N. Caon and I. Trujillo, A Correlation between Galaxy Light Concentration and Supermassive Black Hole Mass, ApJ 563 [Dec., 2001) LI1-L14, [astro-ph/0111152].

[140] A. W. Graham, P. Erwin, N. Caon and I. Trujillo,

A photometric method to determine supermassive black hole masses, in Revista Mexicana de Astronomia y Astrofisica Conference Series (V. Avila-Reese, C. Firmani, C. S. Frenk and C. Allen, eds.), vol. 17 of Revista Mexicana de Astronomia y Astrofisica, vol. 27, pp. 196-197, June, 2003, astro-ph/0206248.

[141] A. W. Graham and S. P. Driver,

A Log-Quadratic Relation for Predicting Supermassive Black Hole Masses from the Host Bulge Sérsic Index, ApJ 655 (Jan., 2007) 77-87, [astro-ph/0607378].

[142] G. Savorgnan, A. W. Graham, A. Marconi, E. Sani, L. K. Hunt, M. Vika et al., The supermassive black hole mass-Sérsic index relations for bulges and elliptical galaxies, MNRAS [34 (Sept., 2013) 387-397, [1306.2679].

[143] G. A. D. Savorgnan,

Supermassive Black Holes and their Host Spheroids III. The Mbh-nsph Correlation, ApJ 821 (Apr. 2016) 88, [11603.01910]. 
[144] M. S. Seigar, D. Kennefick, J. Kennefick and C. H. S. Lacy, Discovery of a Relationship between Spiral Arm Morphology and Supermassive Black Hole Mass in Disk Galaxies, ApJ 678(May, 2008) L93, [0804.0773].

[145] B. L. Davis, J. C. Berrier, D. W. Shields, J. Kennefick, D. Kennefick, M. S. Seigar et al., Measurement of Galactic Logarithmic Spiral Arm Pitch Angle Using Two-dimensional Fast Fourier Transform Decompo ApJS 199 (Apr., 2012) 33, [1202.4780].

[146] J. C. Berrier, B. L. Davis, D. Kennefick, J. D. Kennefick, M. S. Seigar, R. S. Barrows et al., Further Evidence for a Supermassive Black Hole Mass-Pitch Angle Relation, ApJ769 (June, 2013) [132, [1304.4937].

[147] F. Koliopanos, B. C. Ciambur, A. W. Graham, N. A. Webb, M. Coriat, B. Mutlu-Pakdil et al., Searching for intermediate-mass black holes in galaxies with low-luminosity agn: a multiple-method approach, Astronomy \& Astrophysics 601 (2017) A20.

[148] A. Merloni, S. Heinz and T. di Matteo, A Fundamental Plane of black hole activity, MNRAS 345 (Nov., 2003) 1057-1076, [astro-ph/0305261].

[149] H. Falcke, E. Körding and S. Markoff, A scheme to unify low-power accreting black holes. Jet-dominated accretion flows and the radio/X-ray correlation, A\&A 414(Feb., 2004) 895-903, [astro-ph/0305335].

[150] E. Gallo, R. P. Fender and G. G. Pooley, A universal radio-X-ray correlation in low/hard state black hole binaries, MNRAS 344(Sept., 2003) 60-72, [astro-ph/0305231].

[151] R. Narayan, Low-Luminosity Accretion in Black Hole X-Ray Binaries and Active Galactic Nuclei, Ap\&SS 300(Nov., 2005) 177-188, [astro-ph/0411385].

[152] R. P. Fender, J. Homan and T. M. Belloni, Jets from black hole X-ray binaries: testing, refining and extending empirical models for the coupling to X-rays, MNRAS 396 (July, 2009) 1370-1382, [0903.5166].

[153] R. D. Blandford and R. L. Znajek, Electromagnetic extraction of energy from Kerr black holes, MNRAS 179 (May, 1977) 433-456.

[154] R. D. Blandford and D. G. Payne, Hydromagnetic flows from accretion discs and the production of radio jets, MNRAS 199 (June, 1982) 883-903.

[155] M. C. Begelman, R. D. Blandford and M. J. Rees, Theory of extragalactic radio sources, Reviews of Modern Physics 56 (Apr., 1984) 255-351.

[156] C. Done, M. Gierliński and A. Kubota, Modelling the behaviour of accretion flows in X-ray binaries. Everything you always wanted to know about accretion but A\&A Rev. 15(Dec., 2007) 1-66, [0708.0148].

[157] M. Gilfanov, X-Ray Emission from Black-Hole Binaries, in Lecture Notes in Physics, Berlin Springer Verlag (T. Belloni, ed.), vol. 794 of Lecture Notes in Physics, Berlin Springer Verlag, p. 17, Mar., 2010, DOU.

[158] N. I. Shakura and R. A. Sunyaev, Black holes in binary systems. Observational appearance., A\&A 24 (1973) 337-355. 
[159] S. Heinz and R. A. Sunyaev,

The non-linear dependence of flux on black hole mass and accretion rate in core-dominated jets, MNRAS 343 (Aug., 2003) L59-L64, [astro-ph/0305252].

[160] D. L. Meier,

The Association of Jet Production with Geometrically Thick Accretion Flows and Black Hole Rotation, ApJ 548(Feb., 2001) L9-L12, [astro-ph/0010231].

[161] T. J. Maccarone, E. Gallo and R. Fender,

The connection between radio-quiet active galactic nuclei and the high/soft state of X-ray binaries, MNRAS 345 (Oct., 2003) L19-L24, [astro-ph/0309137].

[162] E. G. Körding, S. Jester and R. Fender, Accretion states and radio loudness in active galactic nuclei: analogies with X-ray binaries, MRRAS 372(Nov., 2006) 1366-1378, astro-ph/0608628.

[163] C. A. Jackson and J. V. Wall, Extragalactic radio-source evolution under the dual-population unification scheme, MNRAS 304 (Mar., 1999) 160-174, [astro-ph/9808054].

[164] F. Nicastro, Broad Emission Line Regions in Active Galactic Nuclei: The Link with the Accretion Power, ApJ 530(Feb., 2000) L65-L68, [astro-ph/9912524].

[165] G. Ghisellini and A. Celotti, Relativistic large-scale jets and minimum power requirements, MNRAS 327(Nov., 2001) 739-743, [astro-ph/0103007].

[166] M. Böttcher and C. D. Dermer, An Evolutionary Scenario for Blazar Unification, ApJ 564(Jan., 2002) 86-91, astro-ph/0106395.

[167] M. J. Hardcastle, D. A. Evans and J. H. Croston, The active nuclei of $\mathrm{z}<1.0$ 3CRR radio sources, MNRAS 396 (July, 2009) 1929-1952, [0904.1323].

[168] J. R. Trump, C. D. Impey, B. C. Kelly, F. Civano, J. M. Gabor, A. M. Diamond-Stanic et al., Accretion Rate and the Physical Nature of Unobscured Active Galaxies, ApJ 733 (May, 2011) 60, [1103.0276].

[169] R. M. Plotkin, S. F. Anderson, W. N. Brandt, S. Markoff, O. Shemmer and J. Wu, The Lack of Torus Emission from BL Lacertae Objects: An Infrared View of Unification with WISE, ApJ745(Feb., 2012)L27, [1112.5162].

[170] M. Mezcua, J. Hlavacek-Larrondo, J. R. Lucey, M. T. Hogan, A. C. Edge and B. R. McNamara, The most massive black holes on the Fundamental Plane of black hole accretion, MNRAS 474 [Feb., 2018) 1342-1360, [1710.10268].

[171] A. E. Reines, R. M. Plotkin, T. D. Russell, M. Mezcua, J. J. Condon, G. R. Sivakoff et al., A Candidate Massive Black Hole in the Low-metallicity Dwarf Galaxy Pair Mrk 709, ApJ787 [June, 2014) L30, [1405.0278].

[172] M. Coleman Miller and D. P. Hamilton, Production of intermediate-mass black holes in globular clusters, Monthly Notices of the Royal Astronomical Society 330 (2002) 232-240.

[173] S. Sigurdsson and L. Hernquist, Primordial black holes in globular clusters, Nature 364 (1993) 423-425.

[174] S. Kulkarni, P. Hut and S. J. McMillan, Stellar black holes in globular clusters, Nature 364 (1993) 421-423. 
[175] R. de Vita, M. Trenti and M. MacLeod,

Wandering off the centre: a characterization of the random motion of intermediate-mass black holes in star clusters, MNRAS 475 (Apr., 2018) 1574-1586, [1712.05411]].

[176] S. F. P. Zwart and S. L. McMillan, The runaway growth of intermediate-mass black holes in dense star clusters, The Astrophysical Journal 576 (2002) 899.

[177] S. F. P. Zwart, H. Baumgardt, P. Hut, J. Makino and S. L. McMillan, Formation of massive black holes through runaway collisions in dense young star clusters, Nature $\mathbf{4 2 8}$ (2004) 724-726.

[178] M. Freitag, P. Amaro-Seoane and V. Kalogera, Stellar Remnants in Galactic Nuclei: Mass Segregation, ApJ 649 (Sept., 2006)91-117, [astro-ph/0603280].

[179] L. R. Yungelson, E. P. J. van den Heuvel, J. S. Vink, S. F. Portegies Zwart and A. de Koter, On the evolution and fate of super-massive stars, A\&A 477 (Jan., 2008) 223-237, [0710.1181].

[180] E. Glebbeek, E. Gaburov, S. E. de Mink, O. R. Pols and S. F. Portegies Zwart, The evolution of runaway stellar collision products, A\&A 497 (Apr., 2009) 255-264, [0902.1753].

[181] D. Vanbeveren, H. Belkus, J. van Bever and N. Mennekens, Stellar dynamics in young clusters: the formation of massive runaways and very massive runaway mergers, Ap\&SS 324(Dec., 2009) 271-276, [0712.3343].

[182] J. Bahcall and R. A. Wolf, Star distribution around a massive black hole in a globular cluster, The Astrophysical Journal 209 (1976) 214-232.

[183] R. C. Peterson, P. Seitzer and K. M. Cudworth, The nonthermal stellar dynamics of the globular cluster $\mathrm{m} 15$, The Astrophysical Journal 347 (1989) 251-265.

[184] K. Gebhardt, C. Pryor, T. B. Williams, J. E. Hesser and P. B. Stetson, Fabry-Perot Observations of Globular Clusters.III.M15, AJ113 (Mar., 1997) 1026-1038, [astro-ph/9612116].

[185] K. Gebhardt, C. Pryor, R. D. O'Connell, T. B. Williams and J. E. Hesser, Canada-France-Hawaii Telescope Adaptive Optics Observations of the Central Kinematics in M15, AJ119(Mar., 2000) 1268-1281, [astro-ph/9912172].

[186] K. Gebhardt, R. M. Rich and L. C. Ho, A 20,000 M solar Black Hole in the Stellar Cluster G1, Ap 578(Oct., 2002) L4I-L45, [astro-ph/0209313].

[187] K. Gebhardt, R. M. Rich and L. C. Ho,

An Intermediate-Mass Black Hole in the Globular Cluster G1: Improved Significance from New Keck and Hubble Space ApJ 634(Dec., 2005) 1093-1102, [astro-ph/0508251].

[188] J. Gerssen, R. P. van der Marel, K. Gebhardt, P. Guhathakurta, R. C. Peterson and C. Pryor, Hubble Space Telescope Evidence for an Intermediate-Mass Black Hole in the Globular Cluster M15. II. Kinematic Anal AJ124(Dec., 2002) 3270-3288, [astro-ph/0209315].

[189] E. Noyola, K. Gebhardt and M. Bergmann, Gemini and Hubble Space Telescope Evidence for an Intermediate-Mass Black Hole in $\omega$ Centauri, Ap J 676 (Apr., 2008) 1008-1015, [0801.2782].

[190] E. Noyola, K. Gebhardt, M. Kissler-Patig, N. Lützgendorf, B. Jalali, P. T. de Zeeuw et al., Very Large Telescope Kinematics for Omega Centauri: Further Support for a Central Black Hole, ApJ 719(Aug., 2010) L60-L64, [1007.4559]. 
[191] N. Lützgendorf, M. Kissler-Patig, K. Gebhardt, H. Baumgardt, E. Noyola, B. Jalali et al., Central kinematics of the globular cluster NGC 2808: upper limit on the mass of an intermediate-mass black hole, A\&A 542(June, 2012) A129, [1204.4074]].

[192] N. Lützgendorf, M. Kissler-Patig, K. Gebhardt, H. Baumgardt, E. Noyola, P. T. de Zeeuw et al., Limits on intermediate-mass black holes in six Galactic globular clusters with integral-field spectroscopy, A\&A 552 (Apr., 2013) A49, [1212.3475].

[193] H. Baumgardt, P. Hut, J. Makino, S. McMillan and S. Portegies Zwart, On the Central Structure of M15, ApJ 582 (Jan., 2003) L21-L24, [astro-ph/0210133].

[194] R. van den Bosch, T. de Zeeuw, K. Gebhardt, E. Noyola and G. van de Ven, The Dynamical Mass-to-Light Ratio Profile and Distance of the Globular Cluster M15, ApJ 641 Apr., 2006) 852-86], astro-ph/0512503].

[195] J. Anderson and R. P. van der Marel, New Limits on an Intermediate-Mass Black Hole in Omega Centauri. I. Hubble Space Telescope Photometry and Proper ApJ 710 (Feb., 2010) 1032-1062, [0905.0627].

[196] R. P. van der Marel and J. Anderson, New Limits on an Intermediate-Mass Black Hole in Omega Centauri. II. Dynamical Models, ApJ 710 (Feb., 2010) 1063-1088, [0905.0638].

[197] B. B. P. Perera, B. W. Stappers, A. G. Lyne, C. G. Bassa, I. Cognard, L. Guillemot et al., Evidence for an intermediate-mass black hole in the globular cluster NGC 6624, MNRAS 468 [June, 2017) 2114-2127, [11705.01612].

[198] B. Kiziltan, H. Baumgardt and A. Loeb, An intermediate-mass black hole in the centre of the globular cluster 47 tucanae, Nature 542 (2017) 203-205.

[199] T. J. Maccarone, R. P. Fender and A. K. Tzioumis, Upper limits on central black hole masses of globular clusters from radio emission and a possible black hole détection in MNRAS 356 (Jan., 2005) L17-L22, [astro-ph/0411109].

[200] T. J. Maccarone and M. Servillat, Radio observations of NGC 2808 and other globular clusters: constraints on intermediate-mass black holes, MNRAS 389 (Sept., 2008) 379-384, [0806.2387].

[201] F. N. Bash, K. Gebhardt, W. M. Goss and P. A. Vanden Bout, Very Large Array Limits for Intermediate-Mass Black Holes in Three Globular Clusters, AT135 [Jan., 2008) 182-186, [0710.0339].

[202] D. Cseh, P. Kaaret, S. Corbel, E. Körding, M. Coriat, A. Tzioumis et al., Radio observations of NGC 6388: an upper limit on the mass of its central black hole, MNRAS 406 (Aug., 2010) 1049-1054, [1003.4604].

[203] J. Strader, L. Chomiuk, T. J. Maccarone, J. C. A. Miller-Jones, A. C. Seth, C. O. Heinke et al., No Evidence for Intermediate-mass Black Holes in Globular Clusters: Strong Constraints from the JVLA, ApJ 750(May, 2012) L27, [1203.6352].

[204] J. M. Wrobel, K. E. Nyland and J. C. A. Miller-Jones, A Stacked Search for Intermediate-mass Black Holes in 337 Extragalactic Star Clusters, (Oct., 2015) 120, [1507.07613].

[205] D. Haggard, A. M. Cool, C. O. Heinke, R. Van der Marel, H. N. Cohn, P. M. Lugger et al., A deep chandra X-ray limit on the putative imbh in omega centauri, The Astrophysical Journal Letters 773 (2013) L31. 
[206] D. Pooley and S. Rappaport,

X-Rays from the Globular Cluster G1: Intermediate-Mass Black Hole or Low-Mass X-Ray Binary?, ApJ 644(June, 2006) L45-L48, [astro-ph/0605049].

[207] J. S. Ulvestad, J. E. Greene and L. C. Ho,

Radio Emission from the Intermediate-Mass Black Hole in the Globular Cluster G1, ApJ 661 (June, 2007)L151-L154, [0704.1458].

[208] A. Kong, C. Heinke, R. Di Stefano, H. Cohn, P. Lugger, P. Barmby et al., Localization of the X-ray source in the globular cluster g1 with chandra, Monthly Notices of the Royal Astronomical Society: Letters 407 (2010) L84-L88.

[209] J. C. A. Miller-Jones, J. M. Wrobel, G. R. Sivakoff, C. O. Heinke, R. E. Miller, R. M. Plotkin et al., The Absence of Radio Emission from the Globular Cluster G1, ApJ 755 (Aug., 2012) LI, [1206.5729].

[210] K. Holley-Bockelmann, K. Gültekin, D. Shoemaker and N. Yunes, Gravitational wave recoil and the retention of intermediate-mass black holes, The Astrophysical Journal 686 (2008) 829.

[211] P. Kaaret, H. Feng and T. P. Roberts, Ultraluminous X-Ray Sources, ARA\&A 55 (Aug., 2017) [303-34], [11703.10728].

[212] E. J. M. Colbert and R. F. Mushotzky,

The Nature of Accreting Black Holes in Nearby Galaxy Nuclei, ApJ 519 (July, 1999) 89-107, [astro-ph/9901023].

[213] K. Makishima, A. Kubota, T. Mizuno, T. Ohnishi, M. Tashiro, Y. Aruga et al., The Nature of Ultraluminous Compact X-Ray Sources in Nearby Spiral Galaxies, ApJ 535 (June, 2000) 632-643, [astro-ph/0001009].

[214] P. Kaaret, A. H. Prestwich, A. Zezas, S. S. Murray, D.-W. Kim, R. E. Kilgard et al., Chandra High-Resolution Camera observations of the luminous X-ray source in the starburst galaxy M82, MNRAS 321 (Feb., 2001) L29-L32, [astro-ph/0009211].

[215] J. M. Miller, G. Fabbiano, M. C. Miller and A. C. Fabian, X-Ray Spectroscopic Evidence for Intermediate-Mass Black Holes: Cool Accretion Disks in Two Ultraluminous X-Ray ApJ 585 (Mar., 2003) L37-L40, [astro-ph/0211178].

[216] A. R. King, M. B. Davies, M. Ward, G. Fabbiano and M. Elvis, Ultraluminous x-ray sources in external galaxies, The Astrophysical Journal Letters 552 (2001) L109.

[217] K. Gültekin, M. C. Miller and D. P. Hamilton, Growth of intermediate-mass black holes in globular clusters, The Astrophysical Journal 616 (2004) 221.

[218] K. Gültekin, M. C. Miller and D. P. Hamilton, Three-body dynamics with gravitational wave emission, The Astrophysical Journal 640 (2006) 156.

[219] L. Blecha, N. Ivanova, V. Kalogera, K. Belczynski, J. Fregeau and F. Rasio, Close binary interactions of intermediate-mass black holes: Possible ultraluminous x-ray sources?, The Astrophysical Journal 642 (2006) 427.

[220] R. M. OâĂŹleary, F. A. Rasio, J. M. Fregeau, N. Ivanova and R. OâĂŹShaughnessy, Binary mergers and growth of black holes in dense star clusters, The Astrophysical Journal 637 (2006) 937.

[221] Y. Gao, Q. D. Wang, P. N. Appleton and R. A. Lucas, Nonnuclear Hyper/Ultraluminous X-Ray Sources in the Starbursting Cartwheel Ring Galaxy, ApJ 596 (Oct., 2003) L171-L174, [astro-ph/0309253]. 
[222] M. Gilfanov, H.-J. Grimm and R. Sunyaev, HMXB, ULX and star formation, Nuclear Physics B Proceedings Supplements 132 (June, 2004) 369-375, [astro-ph/0309725].

[223] T. P. Roberts, R. S. Warwick, M. J. Ward and M. R. Goad, Chandra observations of five ultraluminous X-ray sources in nearby galaxies, MNRAS 349 (Apr., 2004)1193-1210, astro-ph/0401306.

[224] J. Poutanen, G. Lipunova, S. Fabrika, A. G. Butkevich and P. Abolmasov, Supercritically accreting stellar mass black holes as ultraluminous X-ray sources, MNRAS 377 (May, 2007) 1187-1194, [astro-ph/0609274].

[225] A. R. King, Masses, beaming and Eddington ratios in ultraluminous X-ray sources, MNRAS 393 [Feb., 2009) L41-L44, [0811, 1473].

[226] A.-M. Stobbart, T. P. Roberts and J. Wilms, XMM-Newton observations of the brightest ultraluminous X-ray sources, MNRAS 368 (May, 2006) B97-413, astro-ph/0601651].

[227] J. C. Gladstone, T. P. Roberts and C. Done, The ultraluminous state, MNRAS 397(Aug., 2009) ए836-185], [0905.4076].

[228] A. D. Sutton, T. P. Roberts and M. J. Middleton, The ultraluminous state revisited: fractional variability and spectral shape as diagnostics of super-Eddington accretion, MNRAS 435 (Oct., 2013) 1758-1775, [1307.8044].

[229] M. J. Middleton, L. Heil, F. Pintore, D. J. Walton and T. P. Roberts, A spectral-timing model for ULXs in the supercritical regime, MNRAS 447 (Mar., 2015) B243-32631, [11412.4532]].

[230] M. Bachetti, F. A. Harrison, D. J. Walton, B. W. Grefenstette, D. Chakrabarty, F. Fürst et al., An ultraluminous X-ray source powered by an accreting neutron star, Nature 514 (Oct., 2014) [202-204, [1410,3590].

[231] G. L. Israel, A. Belfiore, L. Stella, P. Esposito, P. Casella, A. De Luca et al., An accreting pulsar with extreme properties drives an ultraluminous X-ray source in NGC 5907, Science 355 (Feb., 2017) 817-819, [11609.07375]].

[232] F. Fürst, D. J. Walton, F. A. Harrison, D. Stern, D. Barret, M. Brightman et al., Discovery of Coherent Pulsations from the Ultraluminous X-Ray Source NGC 7793 P13, ApJ 831] (Nov., 2016) L14, [1609.07129].

[233] A. King and J.-P. Lasota, ULXs: Neutron stars versus black holes, MNRAS 458 (May, 2016) [10-12], [1601.03738].

[234] A. King, J.-P. Lasota and W. Kluźniak, Pulsing ULXs: tip of the iceberg?, MNRAS 468 (June, 2017) [L59-162, [1702.00808].

[235] A. A. Mushtukov, V. F. Suleimanov, S. S. Tsygankov and A. Ingram, Optically thick envelopes around ULXs powered by accreating neutron stars, MNRAS (Jan., 2017), [1612.00964].

[236] F. Koliopanos, G. Vasilopoulos, O. Godet, M. Bachetti, N. A. Webb and D. Barret, ULX spectra revisited: Accreting, highly magnetized neutron stars as the engines of ultraluminous X-ray sources, ArXiv e-prints (Oct., 2017), [11710.04953]. 
[237] G. C. Dewangan, L. Titarchuk and R. E. Griffiths, Black Hole Mass of the Ultraluminous X-Ray Source M82 X-1, ApJ637 (Jan., 2006) L21-L24, [astro-ph/0509646].

[238] S. A. Farrell, N. A. Webb, D. Barret, O. Godet and J. M. Rodrigues,

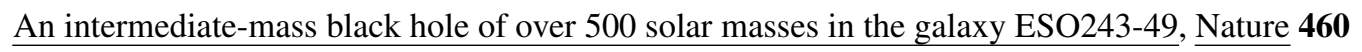
[July, 2009) 73-75, [1001.0567].

[239] M. Servillat, S. A. Farrell, D. Lin, O. Godet, D. Barret and N. A. Webb, X-Ray Variability and Hardness of ESO 243-49 HLX-1: Clear Evidence for Spectral State Transitions, ApJ 743 (Dec., 2011)6, [1108.4405].

[240] N. Webb, D. Cseh, E. Lenc, O. Godet, D. Barret, S. Corbel et al., Radio Detections During Two State Transitions of the Intermediate-Mass Black Hole HLX-1, Science 337 (Aug., 2012) 554, [1311.6918].

[241] H. Feng and P. Kaaret, Identification of the X-ray Thermal Dominant State in an Ultraluminous X-ray Source in M82, ApJ 712 (Apr., 2010) L169-L173, [1003.0283].

[242] M. G. Watson, A. C. Schröder, D. Fyfe, C. G. Page, G. Lamer, S. Mateos et al., The XMM-Newton serendipitous survey. V. The Second XMM-Newton serendipitous source catalogue, A\&A 493 (Jan., 2009) 339-373, [0807.1067].

[243] N. A. Webb, D. Barret, O. Godet, M. Servillat, S. A. Farrell and S. R. Oates, Chandra and Swift Follow-up Observations of the Intermediate-mass Black Hole in ESO 243-49, ApJ712(Mar., 2010) L107-L110, [1002 3625].

[244] R. Soria, G. K. T. Hau, A. W. Graham, A. K. H. Kong, N. P. M. Kuin, I.-H. Li et al., Discovery of an optical counterpart to the hyperluminous X-ray source in ESO 243-49, MNRAS 405 (June, 2010) 870-876, [0910.1356].

[245] K. Wiersema, S. A. Farrell, N. A. Webb, M. Servillat, T. J. Maccarone, D. Barret et al., A Redshift for the Intermediate-mass Black Hole Candidate HLX-1: Confirmation of its Association with the Galaxy ES ApJ721(Oct., 2010) L102-L106, [1008.4125].

[246] R. Soria, G. K. Hau and M. W. Pakull, Kinematics of the intermediate-mass black hole candidate hlx-1, The Astrophysical Journal Letters 768 (2013) L22.

[247] J.-P. Lasota, T. Alexander, G. Dubus, D. Barret, S. Farrell, N. Gehrels et al., The origin of variability of the intermediate-mass black-hole ulx system hlx-1 in eso 243-49, The Astrophysical Journal 735 (2011) 89.

[248] K. Wiersema, S. A. Farrell, N. A. Webb, M. Servillat, T. J. Maccarone, D. Barret et al., A redshift for the intermediate-mass black hole candidate hlx-1: Confirmation of its association with the galaxy eso 243-49, The Astrophysical Journal Letters 721 (2010) L102.

[249] N. A. Webb, A. Guérou, B. Ciambur, A. Detoeuf, M. Coriat, O. Godet et al., Understanding the environment around the intermediate mass black hole candidate ESO 243-49 HLX-1, A\&A 602 (June, 2017) A103, [11704.04434].

[250] O. Godet, B. Plazolles, T. Kawaguchi, J.-P. Lasota, D. Barret, S. A. Farrell et al., Investigating Slim Disk Solutions for HLX-1 in ESO 243-49, ApJ 752 (June, 2012) 34, [1204.346]]. 
[251] S. W. Davis, R. Narayan, Y. Zhu, D. Barret, S. A. Farrell, O. Godet et al.,

The Cool Accretion Disk in ESO 243-49 HLX-1: Further Evidence of an Intermediate-mass Black Hole, ApJ 734(June, 2011)111, [1104.2614]].

[252] O. Straub, O. Godet, N. Webb, M. Servillat and D. Barret, Investigating the mass of the intermediate mass black hole candidate HLX-1 with the slimbh model, A\&A 569(Sept., 2014) A116, [11403.6407].

[253] C. T. Berghea, K. A. Weaver, E. J. M. Colbert and T. P. Roberts, Testing the Paradigm that Ultraluminous X-Ray Sources as a Class Represent Accreting Intermediate-Mass Black Holes, ApJ 687 (Nov., 2008) 471-487, [0807.1547].

[254] F. Grisé, P. Kaaret, H. Feng, J. J. E. Kajava and S. A. Farrell, X-ray Spectral State is not Correlated with Luminosity in Holmberg II X-1, ApJ 724 (Dec., 2010) [148-152], [1011.2231].

[255] M. Brightman, F. A. Harrison, D. Barret, S. W. Davis, F. Fürst, K. K. Madsen et al., A Broadband X-Ray Spectral Study of the Intermediate-mass Black Hole Candidate M82 X-1 with NuSTAR, Chandra, a ApJ 829(Sept., 2016) 28, [1607.03903].

[256] M. Mezcua, T. P. Roberts, A. D. Sutton and A. P. Lobanov, Radio observations of extreme ULXs: revealing the most powerful ULX radio nebula ever or the jet of an intermediate-m MNRAS 436 (Dec., 2013) 3128-3134, [1309.5721].

[257] M. Mezcua, T. P. Roberts, A. P. Lobanov and A. D. Sutton, The powerful jet of an off-nuclear intermediate-mass black hole in the spiral galaxy NGC 2276, MNRAS 448 (Apr., 2015) 1893-1899, [1501.04897].

[258] M. Kim, L. C. Ho, J. Wang, G. Fabbiano, S. Bianchi, M. Cappi et al., An Off-nucleus Nonstellar Black Hole in the Seyfert Galaxy NGC 5252, ApJ 814(Nov., 2015) 8, [1510.08854].

[259] M. Kim, L. C. Ho and M. Im, Ionized Gas Kinematics around an Ultra-luminous X-Ray Source in NGC 5252: Additional Evidence for an Off-nuclear ApJ 844(Aug., 2017) L21, [1707.05402].

[260] D. Merritt and L. Ferrarese, Black hole demographics from the mâŮ́- $\sigma$ relation, Monthly Notices of the Royal Astronomical Society 320 (2001) L30-L34.

[261] A. V. Filippenko, L. C. Ho and W. L. Sargent, Hst observations of ngc 4395, the least luminous seyfert 1 nucleus-evidence against the starburst hypothesis for broad-lined active galactic nuclei, The Astrophysical Journal 410 (1993) L75-L78.

[262] L. C. Ho and J. S. Ulvestad, Radio continuum survey of an optically selected sample of nearby seyfert galaxies, The Astrophysical Journal Supplement Series 133 (2001) 77.

[263] J. Wrobel and L. Ho, Radio emission on subparsec scales from the intermediate-mass black hole in ngc 4395, The Astrophysical Journal Letters 646 (2006) L95.

[264] E. C. Moran, A. V. Filippenko, L. C. Ho, J. C. Shields, T. Belloni, A. Comastri et al., The nuclear spectral energy distribution of ngc 4395, the least luminous type 1 seyfert galaxy, Publications of the Astronomical Society of the Pacific 111 (1999) 801.

[265] D. C. Shih, K. Iwasawa and A. Fabian, Evidence for an intermediate-mass black hole and a multi-zone warm absorber in ngc 4395, Monthly Notices of the Royal Astronomical Society 341 (2003) 973-980. 
[266] S. Vaughan, K. Iwasawa, A. Fabian and K. Hayashida, The exceptional x-ray variability of the dwarf seyfert nucleus ngc 4395, Monthly Notices of the Royal Astronomical Society 356 (2005) 524-530.

[267] E. C. Moran, M. Eracleous, K. M. Leighly, G. Chartas, A. V. Filippenko, L. C. Ho et al., Extreme X-ray behavior of the low-luminosity active nucleus in ngc 4395, The Astronomical Journal 129 (2005) 2108.

[268] E. Nardini and G. Risaliti, The effects of x-ray absorption variability in ngc 4395, Monthly Notices of the Royal Astronomical Society 417 (2011) 2571-2576.

[269] A. V. Filippenko and L. C. Ho, A low-mass central black hole in the bulgeless seyfert 1 galaxy ngc 4395, The Astrophysical Journal Letters 588 (2003) L13.

[270] A. L. King, J. M. Miller, M. T. Reynolds, K. Gültekin, E. Gallo and D. Maitra, A distinctive disk-jet coupling in the lowest-mass seyfert, ngc 4395, The Astrophysical Journal Letters 774 (2013) L25.

[271] S. Corbel, M. Nowak, R. P. Fender, A. K. Tzioumis and S. Markoff, Radio/x-ray correlation in the low/hard state of gx 339-4, Astronomy \& Astrophysics 400 (2003) 1007-1012.

[272] S. Corbel, M. Coriat, C. Brocksopp, A. Tzioumis, R. Fender, J. Tomsick et al., The âĂŸuniversalâĂŹradio/x-ray flux correlation: the case study of the black hole gx 339- 4, Monthly Notices of the Royal Astronomical Society 428 (2012) 2500-2515.

[273] E. Gallo, R. P. Fender and G. G. Pooley, A universal radio-x-ray correlation in low/hard state black hole binaries, Monthly Notices of the Royal Astronomical Society 344 (2003) 60-72.

[274] E. Gallo, B. P. Miller and R. Fender, Assessing luminosity correlations via cluster analysis: Evidence for dual tracks in the radio/x-ray domain of black hole x-ray binaries, Monthly Notices of the Royal Astronomical Society 423 (2012) 590-599.

[275] D. D. Nguyen, A. C. Seth, M. Den Brok, N. Neumayer, M. Cappellari, A. J. Barth et al., Improved dynamical constraints on the mass of the central black hole in ngc 404, The Astrophysical Journal 836 (2017) 237.

[276] K. Nyland, J. Marvil, J. Wrobel, L. M. Young and B. A. Zauderer, The intermediate-mass black hole candidate in the center of ngc 404: New evidence from radio continuum observations, The Astrophysical Journal 753 (2012) 103.

[277] C. E. Thornton, A. J. Barth, L. C. Ho, R. E. Rutledge and J. E. Greene, The host galaxy and central engine of the dwarf active galactic nucleus pox 52, The Astrophysical Journal 686 (2008) 892.

[278] A. W. Graham, B. C. Ciambur and R. Soria,

Does the Intermediate-Mass Black Hole in LEDA 87300 (RGG 118) Follow the Near-quadratic $\mathrm{M}_{B H} \sim \mathrm{M}_{S \text { pheroid }}$ Relation ApJ 818(Feb., 2016) 172, [1512.00991].

[279] V. F. Baldassare, A. E. Reines, E. Gallo and J. E. Greene, A 50,000 $m_{\odot}$ solar mass black hole in the nucleus of rgg 118, The Astrophysical Journal Letters 809 (2015) L14.

[280] M. Mezcua, F. Civano, G. Fabbiano, T. Miyaji and S. Marchesi, A Population of Intermediate-mass Black Holes in Dwarf Starburst Galaxies Up to Redshift=1.5, ApJ 817(Jan., 2016) 20, [1511.05844].

[281] M. Mezcua, F. Civano, S. Marchesi, H. Suh, G. Fabbiano and M. Volonteri, Intermediate-mass black holes in dwarf galaxies out to redshift $\sim 2.4$ in the Chandra COSMOS Legacy Survey, ArXiv e-prints (Feb., 2018), [1802.01567]. 
[282] H. Baumgardt, N-body modelling of globular clusters: masses, mass-to-light ratios and intermediate-mass black holes, Monthly Notices of the Royal Astronomical Society 464 (2016) 2174-2202.

[283] D. E. McLaughlin, J. Anderson, G. Meylan, K. Gebhardt, C. Pryor, D. Minniti et al., Hubble space telescope proper motions and stellar dynamics in the core of the globular cluster 47 tucanae, The Astrophysical Journal Supplement Series 166 (2006) 249.

[284] L. L. Watkins, R. P. van der Marel, A. Bellini and J. Anderson, Hubble space telescope proper motion (hstpromo) catalogs of galactic globular clusters. ii. kinematic profiles and maps, The Astrophysical Journal 803 (2015) 29.

[285] A. Ridolfi, P. C. C. Freire, P. Torne, C. O. Heinke, M. van den Berg, C. Jordan et al., Long-term observations of the pulsars in 47 Tucanae - I. A study of four elusive binary systems, MNRAS 462 (Nov., 2016) 2918-2933, [1607.07248].

[286] P. C. C. Freire, A. Ridolfi, M. Kramer, C. Jordan, R. N. Manchester, P. Torne et al., Long-term observations of the pulsars in 47 Tucanae - II. Proper motions, accelerations and jerks, MNRAS 471 (Oct., 2017) 857-876, [1706.04908].

[287] J. E. Greene and L. C. Ho, A new sample of low-mass black holes in active galaxies, The Astrophysical Journal 670 (2007) 92.

[288] L. C. Ho and M. Kim, Low-mass active galactic nuclei with rapid x-ray variability, The Astrophysical Journal 821 (2016) 48. 